\title{
Mitochondrial "power" drives tamoxifen resistance: NQO1 and GCLC are new therapeutic targets in breast cancer
}

\author{
Marco Fiorillo ${ }^{1,2}$, Federica Sotgia ${ }^{3}$, Diego Sisci ${ }^{1}$, Anna Rita Cappello ${ }^{1}$ and Michael \\ P. Lisanti ${ }^{3}$ \\ ${ }^{1}$ The Department of Pharmacy, Health and Nutritional Sciences, The University of Calabria, Cosenza, 87100, Italy \\ 2 The Paterson Institute, University of Manchester, Withington, M20 4BX, United Kingdom \\ ${ }^{3}$ Translational Medicine, School of Environment and Life Sciences, Biomedical Research Centre, University of Salford, Greater \\ Manchester, M5 4WT, United Kingdom
}

Correspondence to: Anna Rita Cappello, email: annarita.cappello@unical.it

Michael P. Lisanti, email: michaelp.lisanti@gmail.com

Keywords: tamoxifen resistance; endocrine therapy; mitochondria; drug resistance; breast cancer

Received: January 17, 2017

Accepted: February 01, 2017

Published: March 02, 2017

\section{ABSTRACT}

Here, we identified two new molecular targets, which are functionally sufficient to metabolically confer the tamoxifen-resistance phenotype in human breast cancer cells. Briefly, 20 proteins were first selected as potential candidates, based on unbiased proteomics analysis, using tamoxifen-resistant cell lines. Then, the cDNAs of the most promising candidates were systematically transduced into MCF-7 cells. Remarkably, NQO1 and GCLC were both functionally sufficient to autonomously confer a tamoxifen-resistant metabolic phenotype, characterized by i) increased mitochondrial biogenesis, ii) increased ATP production and iii) reduced glutathione levels. Thus, we speculate that pharmacological inhibition of NQO1 and GCLC may be new therapeutic strategies for overcoming tamoxifen-resistance in breast cancer patients. In direct support of this notion, we demonstrate that treatment with a known NQO1 inhibitor (dicoumarol) is indeed sufficient to revert the tamoxifenresistance phenotype. As such, these findings could have important translational significance for the prevention of tumor recurrence in $\operatorname{ER}(+)$ breast cancers, which is due to an endocrine resistance phenotype. Importantly, we also show here that NQO1 has significant prognostic value as a biomarker for the prediction of tumor recurrence. More specifically, higher levels of NQO1 mRNA strongly predict patient relapse in high-risk ER(+) breast cancer patients receiving endocrine therapy (mostly tamoxifen; H.R. > 2.15; $p=0.007$ ).

\section{INTRODUCTION}

Drug-resistance, and the resulting treatment failure, are still significant clinical barriers, preventing more effective cancer therapy and better clinical outcomes [1]. For example, in ER $(+)$ breast cancer, nearly $30 \%$ to $50 \%$ of these patients develop resistance to endocrine-based therapies, such as tamoxifen [2-4]. Tamoxifen-resistance reveals itself clinically as tumor recurrence or systemic metastasis, leading to advanced disease states and premature patient deaths [5]. Thus, endocrine-therapy resistance is a major determinant that significantly reduces the effectiveness of breast cancer therapy. As such, more mechanistic studies are needed to understand the molecular basis of tamoxifen-resistance at the molecular level [6, 7]. Importantly, once these key drivers of the tamoxifenresistance phenotype are identified they may also serve as therapeutic targets and predictive biomarkers to identify high-risk patients, before they become tamoxifen-resistant $[8,9]$.

Here, using a combination of proteomics analysis and metabolic phenotyping, we identified enhanced mitochondrial function and oxidative stress, as key drivers of tamoxifen-resistance [10,11]. In this context, we showed that NQO1 and GCLC, were sufficient to genetically confer tamoxifen-resistance in otherwise tamoxifen-sensitive MCF-7 cells, by enhancing their mitochondrial function $[12,13]$. Thus, NQO1 and GCLC 
may be i) new prognostic biomarkers, ii) novel therapeutic targets and iii) companion diagnostics, for predicting and overcoming tamoxifen-resistance in different subsets of $\mathrm{ER}(+)$ breast cancer patients $[14,15]$.

\section{RESULTS}

\section{Characterization of the metabolic phenotype of tamoxifen-resistant MCF-7 cell lines}

In order to dissect the molecular basis of tamoxifenresistance that occurs during endocrine-based breast cancer therapy, we used tamoxifen-resistant MCF-7 cell lines, such TAMR. The TAMR cell line is serially passaged in the presence of tamoxifen to maintain its resistance phenotype.

First, we validated the tamoxifen-resistance phenotype of TAMR cells. Importantly, TAMR cells behaved in a tamoxifen-resistant manner and continued to grow, despite the presence of increasing concentrations of tamoxifen [16] (Figure 1)

Then, TAMR cells were subjected to metabolic phenotyping in order to establish their behavior. For this purpose, we employed the Seahorse XF96 Analyzer to measure metabolic flux $[17,18]$. Interestingly, Figure 2 illustrates that TAMR cells show an enhanced metabolic phenotype, with significant increases in oxidative mitochondrial metabolism and ATP production, as well as increased basal and maximal respiratory capacity. However, no significant increases in glycolytic rates were observed (Figure 3). This observed increase in ATP production was independently validated using a second independent biochemical assay (Figure 4A). Consistent with the idea that increased mitochondrial metabolism should lead to oxidative stress, we also found that the steady-state levels of reduced glutathione were significantly depleted (Figure 4B). Importantly, enhanced oxidative mitochondrial metabolism was also observed in a second independently-derived tamoxifen-resistant MCF7 cell line, known as TAMR2 (Supplementary Figure 1).

We hypothesized that the observed increase in oxygen consumption and ATP production might occur via an increased capacity for mitochondrial biogenesis. In direct support of our hypothesis, TAMR cells showed a significant increase in both mitochondrial mass and mitochondrial membrane potential (Figure 5), as observed

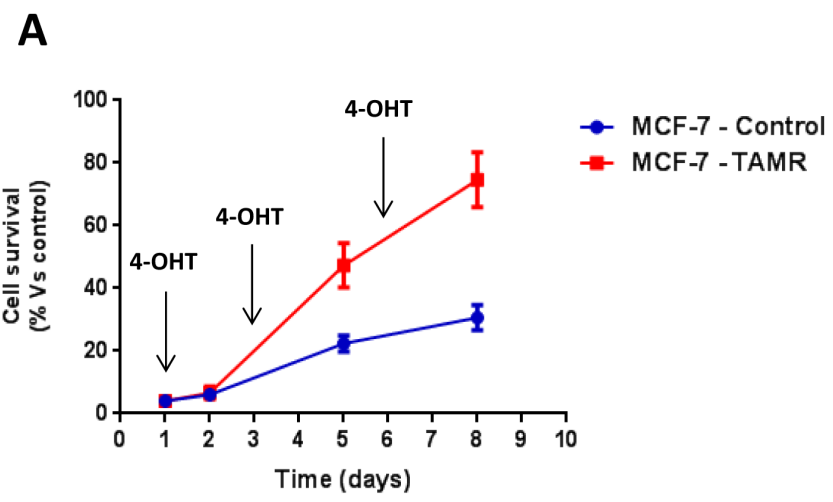

B
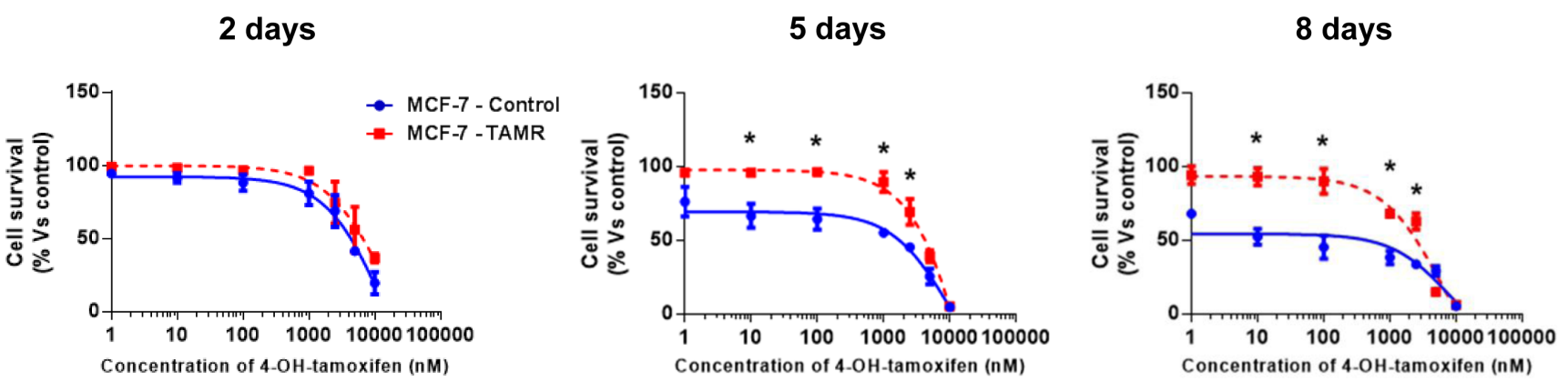

Figure 1: Validation of tamoxifen-resistance in TAMR cells. A. Growth curves of MCF-7-control (WT) and MCF-7-TAMR cells in the presence of 4-OH-Tamoxifen (4-OHT) 100nM. The arrows indicate the day that fresh growth media containing 4-OHT was added to the cell cultures. B. Growth responses of MCF-7-control and MCF-7-TAMR cells to increasing concentrations of 4-OHT (0.1 nM-10 $\mu \mathrm{M})$ on day 2, 5 and 8 from initial treatment. The results for the graphs are expressed as the mean (+/- SD) of six wells repeated three times. * $p<0.01$. 
by FACS analysis using MitoTracker vital dyes as probes (Deep Red and Orange) [19, 20]. However, this phenotype was strictly dependent on the presence of tamoxifen in the tissue culture media.

\section{Proteomics Analysis of TAMR Cells: NQO1 and GCLC are strongly up-regulated}

To begin to dissect the molecular basis of this enhanced metabolic phenotype, TAMR cells were next subjected to unbiased proteomics analysis [21]. This would allow us to identify potential therapeutic targets that might confer tamoxifen-resistance. These results are summarized in Table 1 . We focused on the top 20 proteins that were over-expressed in TAMR cells, relative to matched parental MCF-7 cells.

Note that several of the up-regulated proteins are metabolic enzymes, as predicted. In this data set, we also noticed that the BCAS1 protein was upregulated by $>50$ fold. Importantly, BCAS1 has been previously implicated

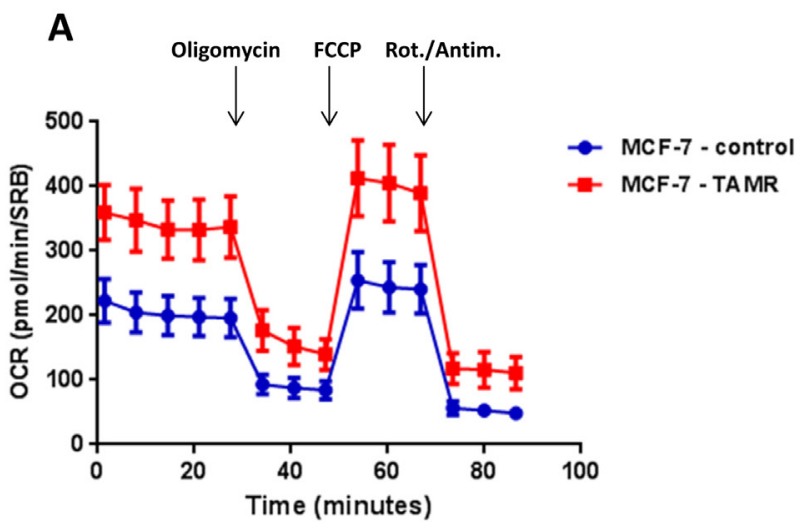

B

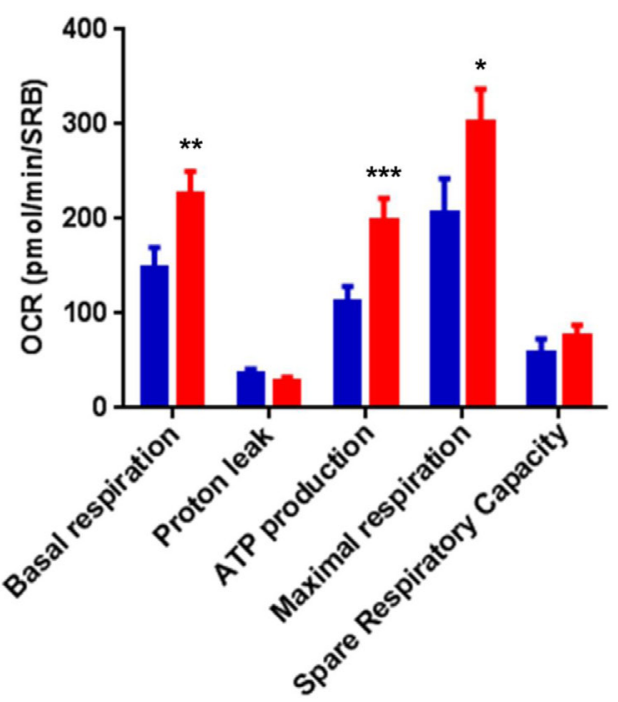

Figure 2: TAMR cells show a significant increase in mitochondrial oxygen consumption and mitochondrial ATP production. The Seahorse XF96 analyzer was employed to determine the mitochondrial function of MCF-7-control cells and MCF-7TAMR after 48 hours. A. A representative line graph of 3 independent experiments is shown. B. Respiration (basal and maximal), as well as ATP levels, were significantly increased.* $p<0.05 ; * * p<0.005 ; * * * p<0.0005$.

A

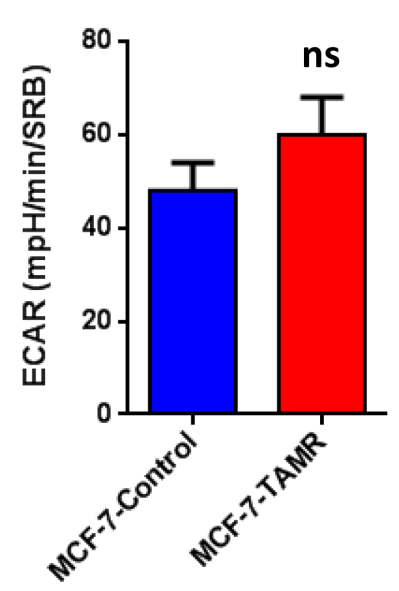

B

\section{OCR Vs ECAR}

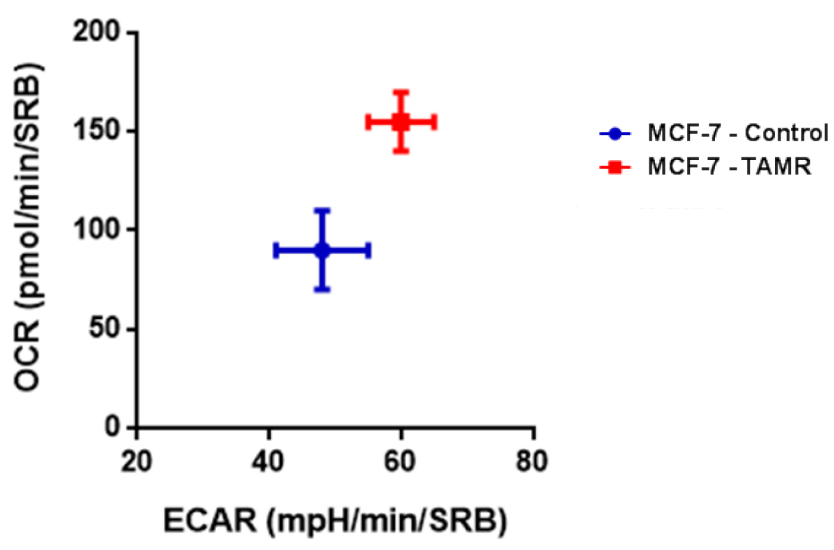

Figure 3: TAMR cells are more energetically active, but do not show any increases in their glycolytic rate. A. The Seahorse XF96 analyzer was employed to determine the status of extracellular acidification rate (ECAR) in MCF-7-control and MCF-7TAMR cells after 48 hours. A bar graph of 3 independent experiments is shown. ns $=$ not significant. B. The plot of OCR versus ECAR shows that MCF-7-TAMR cells shift from a moderate quiescent state to a high energetic state. 
Table 1: List of top 20 proteins up-regulated in tamoxifen-resistant MCF7 cells (MCF-7-TAMR)

$\begin{array}{lll}\text { Symbol } & \text { Description } & \text { Fold-Incri } \\ & & \text { (Up-regul } \\ \text { GPATCH8 } & \text { G patch domain-containing protein 8 } & \text { Infinity } \\ \text { SRRM2 } & \text { Serine/arginine repetitive matrix protein 2 } & \text { Infinity } \\ \text { HSD17B4 } & \text { Peroxisomal multifunctional enzyme type 2 } & 286.23 \\ \text { AKR1C2 } & \text { Aldo-keto reductase family 1 member C2 } & 182.79 \\ \text { NQO1 } & \text { NAD(P)H dehydrogenase [quinone] 1 } & 74.05 \\ \text { BCAS1 } & \text { Breast carcinoma amplified sequence 1 } & 54.13 \\ \text { CTNND1 } & \text { Catenin delta-1 } & 35.23 \\ \text { GCLC } & \text { Glutamate-cysteine ligase } & 29.32 \\ \text { BMP7 } & \text { Bone morphogenetic protein 7 } & 28.74 \\ \text { PI4KA } & \text { Phosphatidylinositol 4-kinase alpha } & 25.59 \\ \text { IDH2 } & \text { Isocitrate dehydrogenase [NADP], mitochondrial } & 22.95 \\ \text { ACAA2 } & \text { 3-ketoacyl-CoA thiolase, mitochondrial } & 22.10 \\ \text { GPCPD1 } & \text { Glycerophosphocholine phosphodiesterase } & 18.01 \\ \text { UGDH } & \text { UDP-glucose 6-dehydrogenase } & 11.31 \\ \text { SMC1A } & \text { Structural maintenance of chromosomes protein 1A } & 11.03 \\ \text { ATP12A } & \text { Potassium-transporting ATPase alpha chain 2 } & 10.89 \\ \text { ATP1B1 } & \text { Sodium/potassium-transporting ATPase subunit beta-1 } & 10.61 \\ \text { STAU1 } & \text { staufen double-stranded RNA binding protein 1 } & 10.03 \\ \text { AHNAK } & \text { Neuroblast differentiation-associated protein } & 9.91 \\ \text { S100P } & \text { Protein S100-P } & 9.76\end{array}$

in conferring tamoxifen-resistance. Thus, we used BCAS1 as a positive control in some of our experiments.

To begin to test our hypothesis that these genes might confer tamoxifen-resistance, we transduced a panel of six candidates into MCF-7 cells (NQO1, GCLC, ACAA2, IDH2, HSD17B4 and BCAS1) and
A

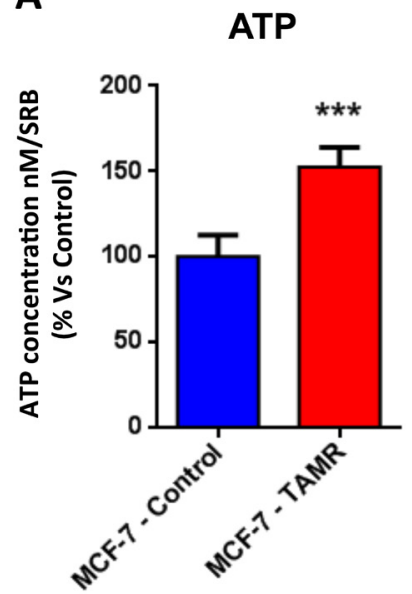

B

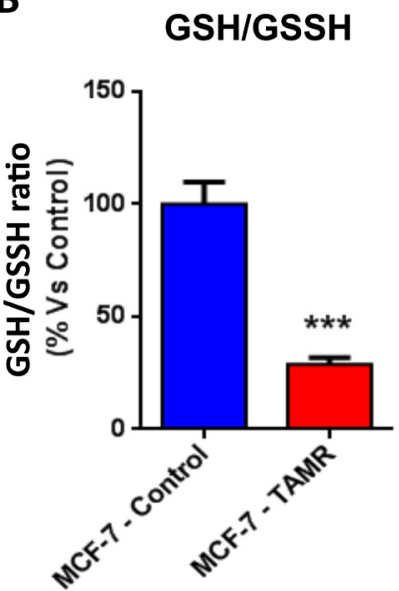

Figure 4: The metabolic phenotype of TAMR cells is characterized by increased steady-state levels of ATP and decreased levels of reduced glutathione. A. ATP levels were evaluated with the Celltiter-Glo ${ }^{\text {TM }}$ luminescent assay kit, after 24 hours of incubation at $37^{\circ} \mathrm{C}$. B. The reduced/oxidized glutathione ratio, was evaluated with the GSH/GSSG-Glo ${ }^{\mathrm{TM}}$ Assay kit, after 24 hours of incubation at $37^{\circ} \mathrm{C}$. Both ATP and glutathion levels were normalized by protein content (SRB) and cell number. $* * * p<0.0005$. 
Mitotracker Deep Red and Orange in TAMR and Control cells in complete media $72 \mathrm{~h}$

A

Mitotracker Deep Red

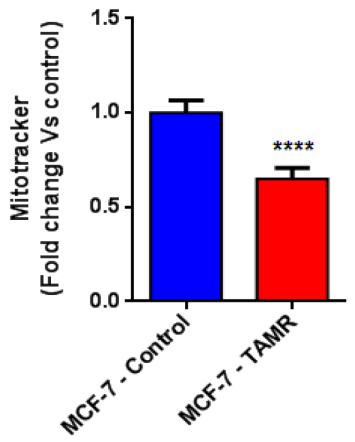

B Mitotracker Orange

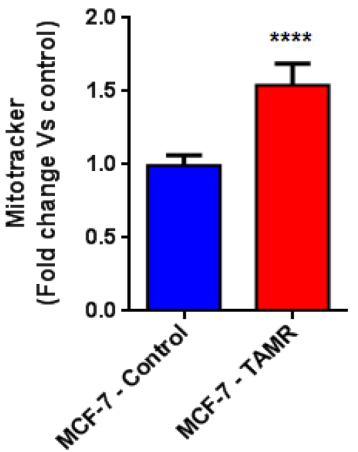

Mitotracker Deep Red and Orange in TAMR Vs Control cells in 4-OHT media 72h

C

Mitotracker Deep Red

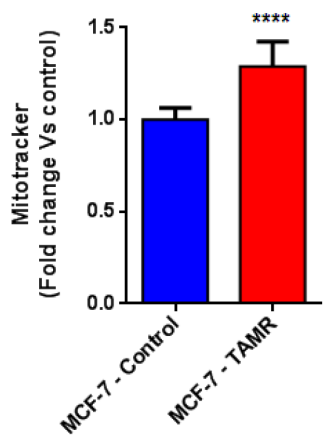

D Mitotracker Orange

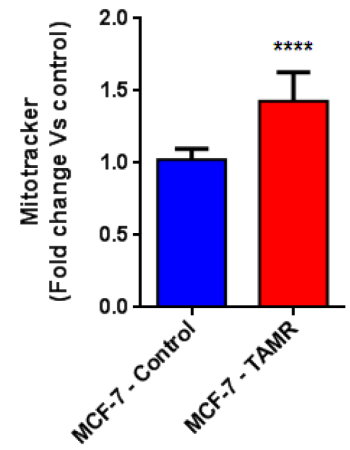

Figure 5: Mitochondrial biogenesis and membrane potential are increased in TAMR cells, in the presence of tamoxifen. FACS analysis was carried out on MCF-7-control and MCF-7-TAMR cells after 72 hours. A.-B. In MCF-7-TAMR, the mitochondrial mass was reduced (MitoTracker Deep-Red), but an increased mitochondrial membrane potential (MitoTracker Orange) was observed after 72 hours of incubation, in growth media 4-OHT free. C.-D. The mitochondrial mass (MitoTracker Deep-Red) and mitochondrial membrane potential (MitoTracker Orange) were increased after 48 hours of incubation, in growth media containing 4-OHT. $* * * * p<0.00001$.

A

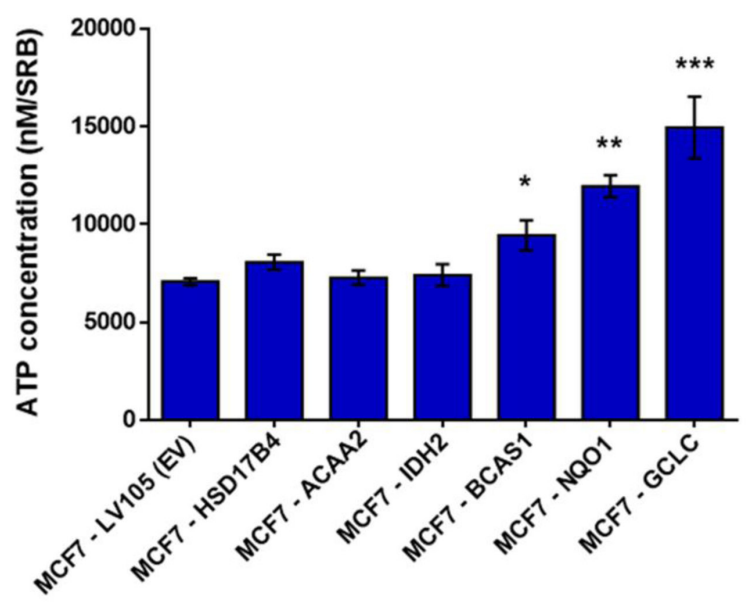

B

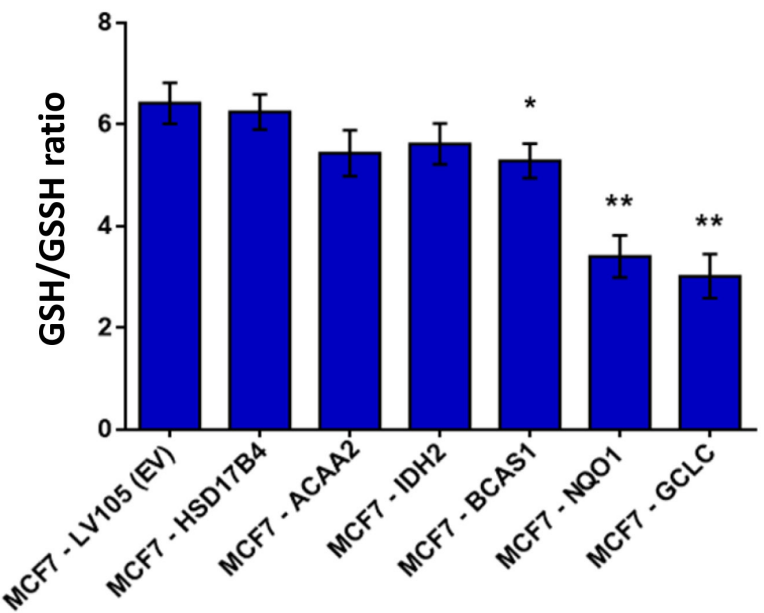

Figure 6: Energetic screening in MCF-7 cells transduced with different cDNAs encoding a subset of proteins upregulated in TAMR cells. A. ATP levels were evaluated with the Celltiter-Glo ${ }^{\mathrm{TM}}$ luminescent assay kit, after 24 hours of incubation at $37^{\circ} \mathrm{C}$. B. The reduced/oxidized glutathione ratio was evaluated with the GSH/GSSG-Glo ${ }^{\mathrm{TM}}$ Assay kit, after 24 hours of incubation at $37^{\circ}$ C. Both ATP and glutathion levels were normalized by protein content (SRB) and cell number. $* p<0.05 ; * * p<0.005$. 
Table 2: List of top 27 proteins up-regulated in MCF-7-NQ01 cells

\begin{tabular}{|c|c|c|}
\hline Symbol & Description & $\begin{array}{l}\text { Fold-Increase } \\
\text { (Up-regulation) }\end{array}$ \\
\hline MED13 & Mediator of RNA polymerase II transcription subunit 13 & Infinity \\
\hline PRDX5 & Peroxiredoxin-5, mitochondrial & 16.99 \\
\hline HSPA4L & Heat shock 70 kDa protein 4L & 16.29 \\
\hline MYO18B & Unconventional myosin-XVIIIb & 10.10 \\
\hline RAB21 & Ras-related protein Rab-21 & 8.74 \\
\hline ADK & Adenosine kinase & 7.60 \\
\hline SQSTM1-ALK & Tyrosine-protein kinase receptor & 3.97 \\
\hline PAMR1 & Inactive serine protease PAMR1 & 3.93 \\
\hline LARP4 & La-related protein 4 & 3.23 \\
\hline BAG3 & BAG family molecular chaperone regulator 3 & 3.21 \\
\hline UCHL5 & Ubiquitin carboxyl-terminal hydrolase L5 & 2.96 \\
\hline KATNAL2 & Katanin p60 ATPase-containing subunit A-like 2 & 2.96 \\
\hline SLC25A3 & Phosphate carrier protein, mitochondrial & 2.91 \\
\hline PKM2 & Pyruvate kinase & 2.82 \\
\hline FPGT & FPGT protein & 2.81 \\
\hline RPS4Y1 & $40 S$ ribosomal protein $S 4, Y$ isoform 1 & 2.79 \\
\hline MRPS22 & $28 \mathrm{~S}$ ribosomal protein $\mathrm{S} 22$, mitochondrial & 2.78 \\
\hline PSMB & Proteasome subunit beta type & 2.77 \\
\hline PRKRA & Interferon-inducible ds-RNA-dependent protein kinase & 2.70 \\
\hline CLEC4C & C-type lectin domain family 4 member $\mathrm{C}$ & 2.66 \\
\hline YWHAE & 14-3-3 protein epsilon & 2.63 \\
\hline KRT14 & Keratin, type I cytoskeletal 14 & 2.46 \\
\hline PAMR1 & Inactive serine protease PAMR1 & 2.39 \\
\hline CEP110 & Centrosomal protein $110 \mathrm{kDa}$ & 2.38 \\
\hline ССT7 & Chaperonin containing TCP1, subunit 7 (Eta) variant & 2.37 \\
\hline CTSD & Cathepsin D & 2.33 \\
\hline GSR & Glutathione reductase, mitochondrial & 2.31 \\
\hline
\end{tabular}

Note that the following 5 mitochondrial and/or metabolism-related enzymes are up-regulated in MCF-7-NQO1 cells (PRDX5, SLC25A3, PKM2, MRPS22, GSR) and are highlighted in BOLD.

then evaluated their metabolic phenotype. As a more efficient screening method, we chose to measure ATP and glutathione levels, to ask which of these candidates would be sufficient to mimic the metabolic phenotype of TAMR cells. As predicted, this screening method was successful and three out of the six candidates (BCAS1, NQO1 and GCLC) actually increased ATP and decreased reduced glutathione (Figure 6). Since NQO1 and GCLC conferred the largest changes in ATP and reduced glutathione, we decided to focus on these two metabolic enzymes as potential mediators of tamoxifen-resistance. NQO1 and GCLC were increased by 74 -fold and by 29 -fold in TAMR cells, respectively, relative to parental MCF-7 cells (Table $1)$.
NQO1 and GCLC are sufficient to confer tamoxifen-resistance and "boost" mitochondrial metabolism

Based on our proteomics analysis and screening approach, NQO1 and GCLC emerged as the most promising potential targets. Thus, we subjected these MCF-7-NQO1 and MCF-7-GCLC cell lines to further characterization. We performed additional mechanistic studies, to determine if they truly mimic the tamoxifenresistance behavior and metabolic phenotype of TAMR cells.

Remarkably, recombinant over-expression of NQO1 
was indeed sufficient to functionally confer tamoxifenresistance (Figure 7). Importantly, empty-vector control MCF-7 cell lines tested in parallel were tamoxifen- sensitive. Similarly, NQO1 over-expression significantly increased oxidative mitochondrial metabolism, with increased levels of ATP production and decreased levels

A

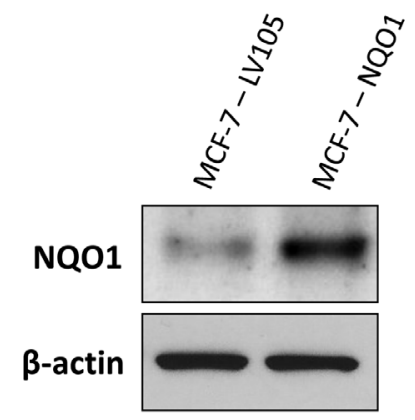

B

2 days

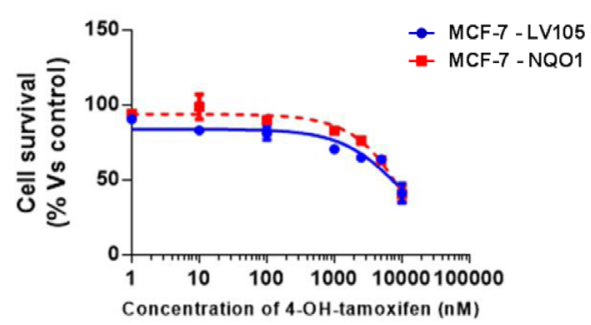

5 days

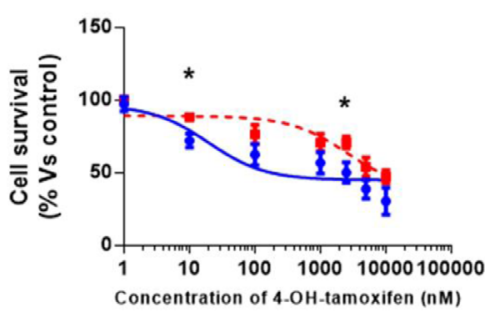

8 days

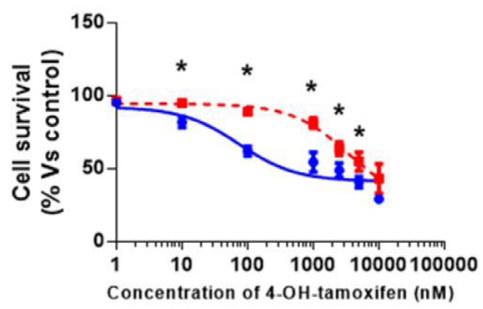

Figure 7: MCF-7 cells over-expressing NQO1 are tamoxifen-resistant. A. MCF-7 cells were stably transduced with the cDNA encoding NQO1 or the empty vector plasmid (LV105-puro), using a lentiviral vector approach. Total cell proteins were isolated from transfected MCF-7 cells and analyzed by immunoblotting to confirm NQO1 expression. NQO1 antibody (\#HPA007308 Sigma-Aldrich) was used with a dilution 1:500 in 5\% of BSA. The expression of $\beta$-actin (\#A2228 Sigma-Aldrich) was also assessed to ensure equal protein loading. B. Growth responses of MCF-7-LV105 and MCF-7-NQO1 cells to increasing concentrations of 4-OHT $(0.1 \mathrm{nM}-10 \mu \mathrm{M})$ on day 2 , 5 and 8 from the initial treatment. The graphs are expressed as the mean (+/-SD) of six wells repeated three times. * $p<0.01$.

A

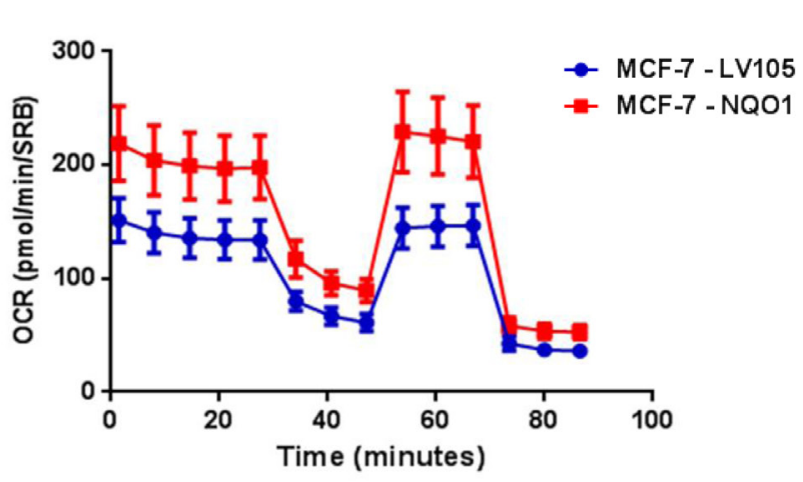

B

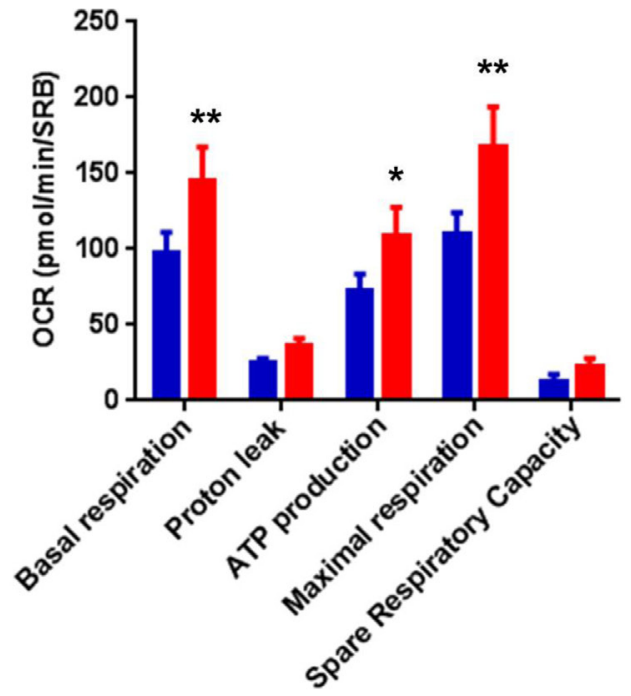

Figure 8: NQO1 expressing MCF7 cells show a significant increase in mitochondrial oxygen consumption and mitochondrial ATP production. The Seahorse XF96 analyzer was employed to determine the status of mitochondrial function in MCF-7-LV105 cells (empty vector) and MCF-7-NQO1 cells after 48 hours. A. A representative line graph of 3 independent experiments is shown. B. Respiration (basal and maximal), as well as ATP levels, were significantly increased. ${ }^{*} p<0.05$; ${ }^{*} p<0.005$. 
Table 3: List of top 29 proteins up-regulated in MCF-7-GCLC cells

\begin{tabular}{|c|c|c|}
\hline Symbol & Description & $\begin{array}{l}\text { Fold-Incre } \\
\text { (Up-regula }\end{array}$ \\
\hline CPSF6 & Cleavage and polyadenylation-specificity factor subunit 6 & Infinity \\
\hline GSPT1 & Eukaryotic peptide chain release factor GTP-binding subunit ERF3A & $5,0127.30$ \\
\hline AlFM1 & Apoptosis-inducing factor 1, mitochondrial & 111.08 \\
\hline DYNC1H1 & Cytoplasmic dynein 1 heavy chain 1 & 109.20 \\
\hline VIM & Vimentin & 55.27 \\
\hline TTN & Titin & 33.27 \\
\hline ATPIF1 & ATPase inhibitor, mitochondrial & 30.89 \\
\hline CYP21A2 & Steroid 21-hydroxylase & 23.49 \\
\hline SERPINH1 & Serpin $\mathrm{H} 1$ & 22.11 \\
\hline KRT4 & Keratin, type II cytoskeletal 4 & 20.88 \\
\hline NOL3 & Nucleolar protein 3 & 20.15 \\
\hline BAG3 & BCL2-associated athanogene 3 & 18.40 \\
\hline CTSD & Cathepsin D & 17.87 \\
\hline HNRNPA2B1 & Heterogeneous nuclear ribonucleoproteins A2/B1 & 17.87 \\
\hline $\mathrm{ZCCHC} 11$ & Terminal uridylyltransferase 4 & 16.47 \\
\hline 2ATP12A & Potassium-transporting ATPase alpha chain & 16.18 \\
\hline YWHAZ & 14-3-3 protein zeta/delta (Fragment) & 15.70 \\
\hline L27a & Ribosomal protein L27a & 14.84 \\
\hline STUB1 & E3 ubiquitin-protein ligase CHIP & 14.55 \\
\hline NCL & Nucleolin & 11.61 \\
\hline G6PD & Glucose-6-phosphate 1-dehydrogenase & 11.25 \\
\hline HSPA4L & Heat shock $70 \mathrm{kDa}$ protein $4 \mathrm{~L}$ & 10.40 \\
\hline FASN & FASN protein & 10.18 \\
\hline PGK2 & Phosphoglycerate kinase 2 & 10.12 \\
\hline UGP2 & UTP--glucose-1-phosphate uridylyltransferase & 9.89 \\
\hline HMGN4 & High mobility group nucleosome-binding domain-containing protein 4 & 9.06 \\
\hline DCXR & L-xylulose reductase & 9.00 \\
\hline CTNNA2 & Catenin alpha-2 & 8.56 \\
\hline ABAT & 4-aminobutyrate aminotransferase, mitochondrial & 7.32 \\
\hline
\end{tabular}

Note that the following 5 mitochondrial and/or metabolism-related enzymes are up-regulated in MCF-7-GCLC cells (AIFM1, ATPIF1, G6PD, PGK2, ABAT) and are highlighted in BOLD.

of reduced glutathione (Figures 8, 9 and 10). Finally, mitochondrial biogenesis and mitochondrial membrane potential were also increased in MCF-7-NQO1 cells (Figure 11).

Importantly, virtually identical results were also obtained with GCLC. MCF-7 cells transduced with the cDNA of GCLC were i) tamoxifen-resistant (Figure 12) and ii) showed a significant increase in mitochondrial oxygen consumption (Figure 13 and 14). Moreover, MCF7-GCLC cells had enhanced ATP production and showed decreased reduced glutathione (Figure 15), with increased mitochondrial biogenesis (Figure 16).

In accordance with the above functional data, a number of mitochondrial and/or other metabolism-related proteins were highly up-regulated, as seen by proteomics analysis, in both NQO1 and GCLC transfected MCF-7 cells (Tables 2 and 3). These up-regulated mitochondrial proteins included ABAT, AIFM1, ATPIF1, GSR, MRPS22 and PRDX5, among others.

In summary, the NQO1 and GCLC genes are both indeed sufficient to confer tamoxifen-resistance and to "boost" mitochondrial metabolism. Conversely, we also observed that treatment of MCF-7-TAMR cells with dicoumarol, a known NQO1 inhibitor, reverses their tamoxifen-resistance phenotype (Supplementary Figures 2 and 3). Thus, pharmacological inhibition of NQO1 and GCLC may be new therapeutic strategies for overcoming tamoxifen-resistance in breast cancer patients. 
Overview of proteins and pathways identified as differentially regulated in the lysates of TAMR, NQO1 and GCLC cells relative to control MCF7 cells and MCF-7-LV105 (empty vector), by ingenuity pathway analysis

From all the proteomics data sets, the differentially expressed proteins were subjected to Ingenuity Pathway Analysis (IPA) to determine possible alterations in canonical pathways and toxicity functions. IPA was able to analyze 1,283 proteins in MCF-7-TAMR, 399 proteins in MCF-7-NQO1 and 684 proteins in MCF-7-GCLC cells, as compared with their respective controls. This analysis revealed that 142 proteins were differentially regulated in all 3 different cell lines (Figure 17A). A higher presence of antioxidant response proteins was further confirmed using IPA, which revealed the Nrf2-mediated antioxidant response to be one of the top canonical pathways affected. Similarly, TAMR, NQO1 and GCLC cells all showed very
A

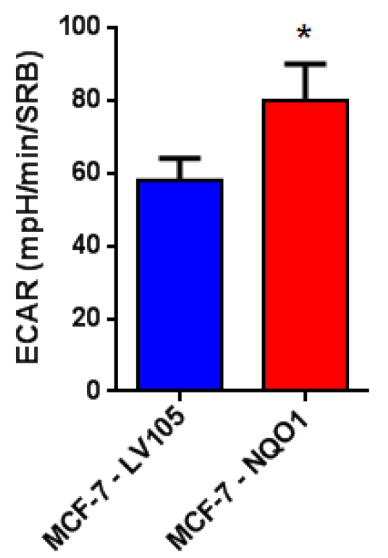

B

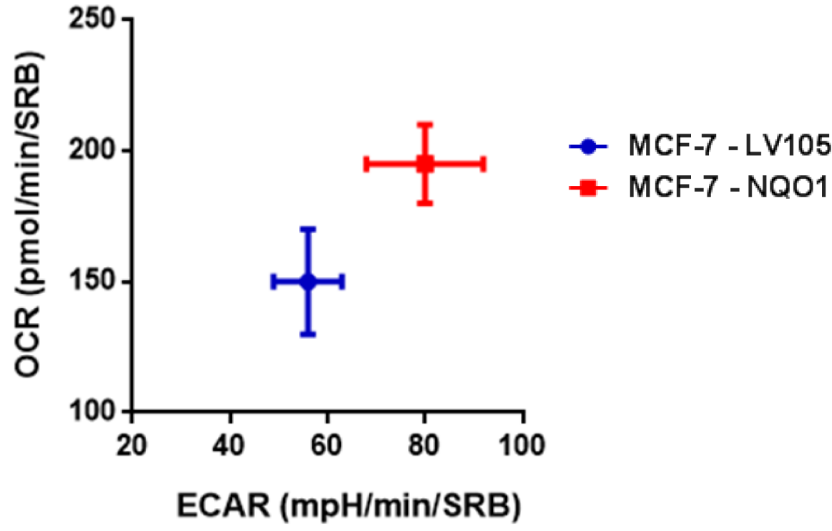

Figure 9: NQO1 expressing MCF7 cells are more energetically active, and show a minor increase in their glycolytic rate. A. The Seahorse XF96 analyzer was employed to determine the status of extracellular acidification rate (ECAR) in MCF-7-LV105 and MCF-7- NQO1 cells after 48 hours. A bar graph of 3 independent experiments is shown. B. The plot of OCR versus ECAR shows that MCF-7-TAMR cells shift from moderate quiescent state to a high energetic state. * $p<0.05$.

A

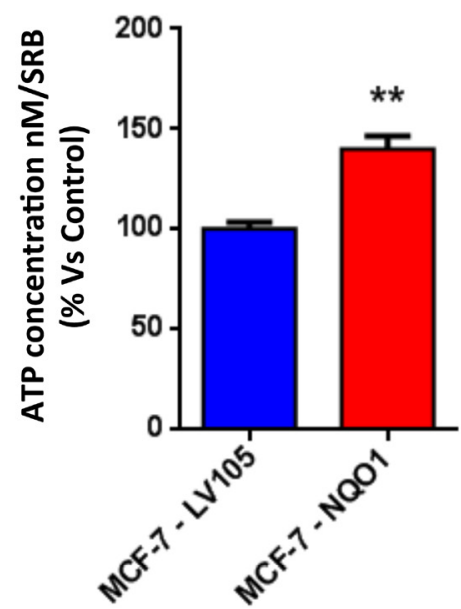

ATP
B

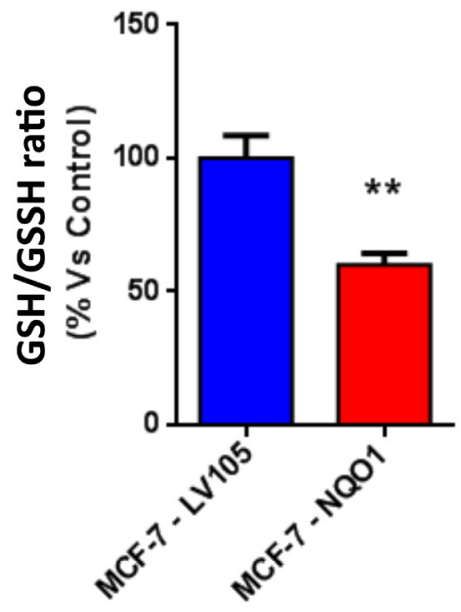

Figure 10: The metabolic phenotype of NQO1 expressing MCF7 cells is characterized by increased steady-state levels of ATP and decreased levels of reduced glutathione. A. ATP concentration levels, evaluate with Celltiter-Glo ${ }^{\mathrm{TM}}$ luminescent assay kit, after 24 hours of incubation at $37^{\circ} \mathrm{C}$. B. Reduced/oxidized glutathione ratio, evaluate with GSH/GSSG-Glo ${ }^{\mathrm{TM}}$ Assay kit, after 24 hours of incubation at $37^{\circ} \mathrm{C}$. Both types of experiments were standardized by protein content (SRB) and cell number. ${ }^{* *} p<0.005$. 


\section{Mitotracker Deep Red and Orange in NQO1 and Control cells in complete media $72 \mathrm{~h}$}

A

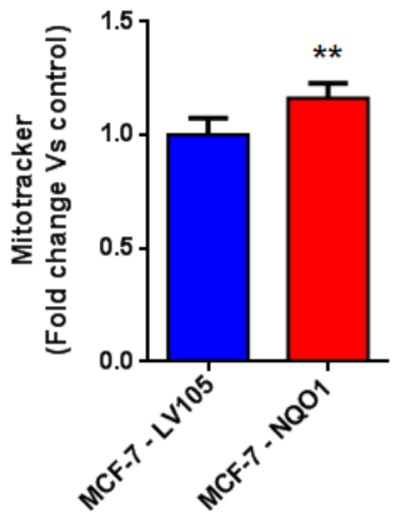

B Mitotracker Orange

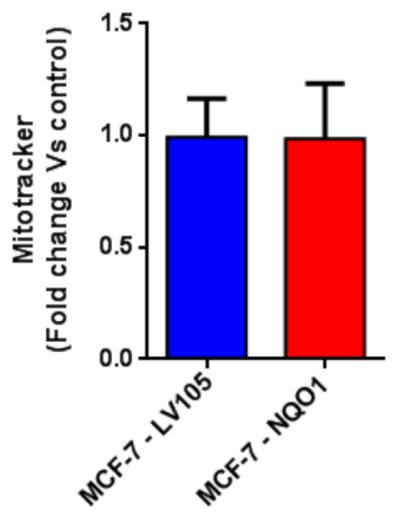

Mitotracker Deep Red and Orange in NQ01 Vs Control cells in 4-OHT media 72h

C

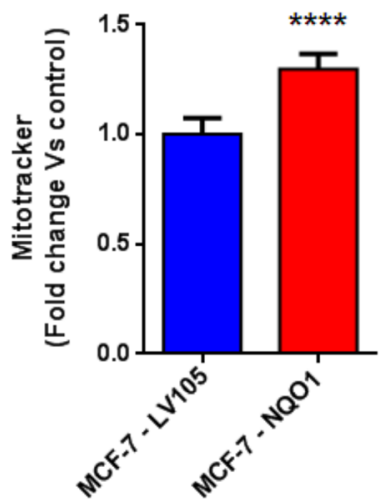

Mitotracker Orange

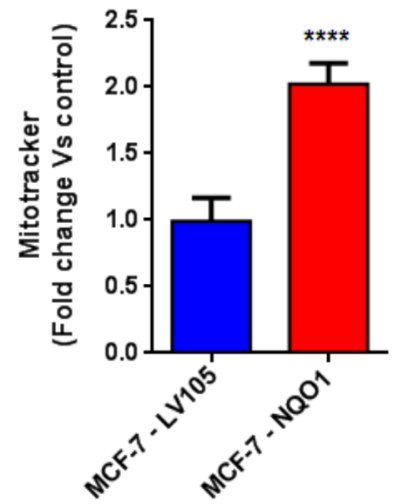

Figure 11: Mitochondrial biogenesis and membrane potential are increased in NQO1 expressing MCF7 cells, in the presence of tamoxifen. FACS analysis was carried out on MCF-7-LV105 (empty vector) and MCF-7-NQO1 after 72 hours. A.-B. In MCF-7-NQO1, the mitochondrial mass was increased (MitoTracker Deep-Red), but not significant changes in mitochondrial membrane potential (MitoTracker Orange) were observed after 72 hours of incubation, in growth media 4-OHT free. C.-D. The mitochondrial mass (MitoTracker Deep-Red) and mitochondrial membrane potential (MitoTracker Orange) were increased after 48 hours of incubation, in growth media containing 4-OHT. $* * p<0.001 ; * * * * p<0.00001$.

\section{2 days}

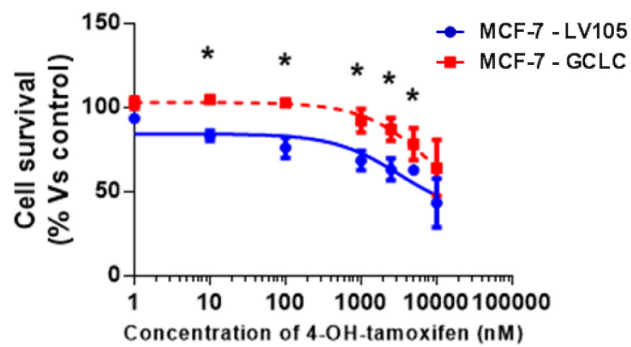

5 days

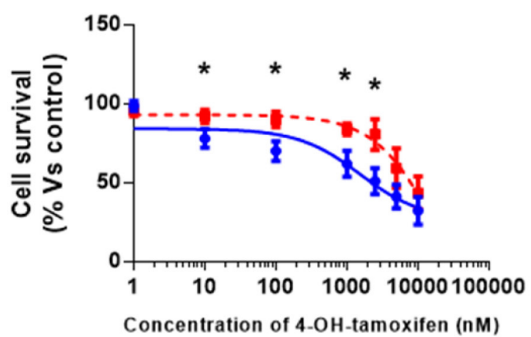

8 days

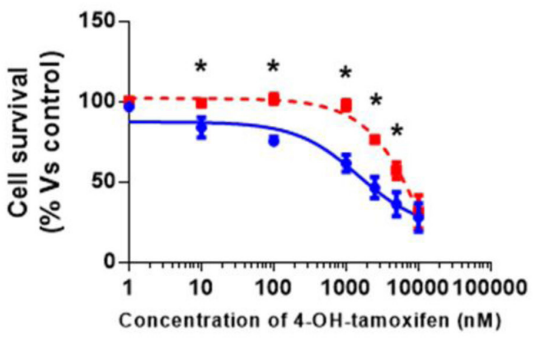

Figure 12: MCF-7 cells harboring GCLC show tamoxifen-resistance. Growth responses of MCF-7-LV105 (empty vector) and MCF-7-GCLC cells to increasing concentrations of 4-OHT $(0.1 \mathrm{nM}-10 \mu \mathrm{M})$ on day 2, 5 and 8 from initial treatment. The results for the graphs are expressed as the mean $(+/-\mathrm{SD})$ of six wells repeated three times. ${ }^{*} p<0.01$. 

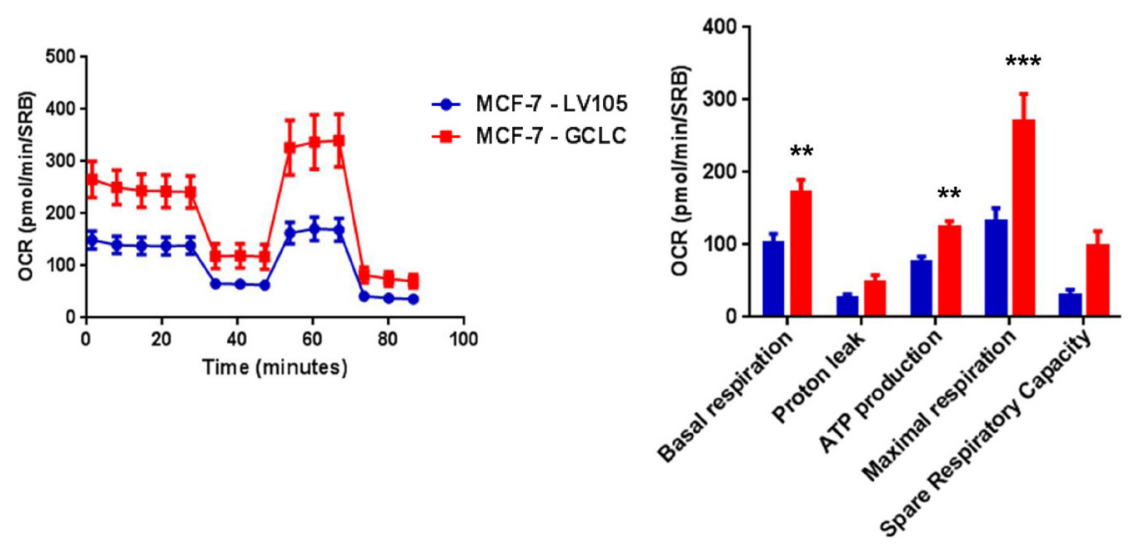

Figure 13: MCF7 cells harboring GCLC show a significant increase in mitochondrial oxygen consumption and mitochondrial ATP production. The Seahorse XF96 analyzer was employed to determine the mitochondrial function of MCF-7LV105 (empty vector) and MCF-7-GCLC cells after 48 hours. A. A representative line graph of 3 independent experiments is shown. B. Respiration (basal and maximal), as well as ATP levels, were significantly increased.** $p<0.005 ; * * * p<0.0005$.

A

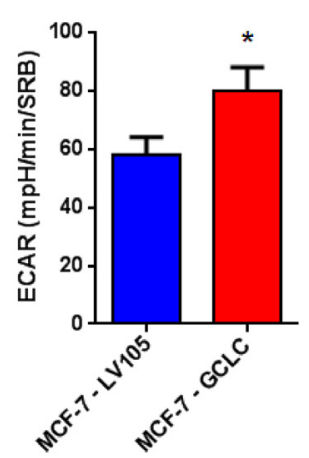

B OCR VS ECAR

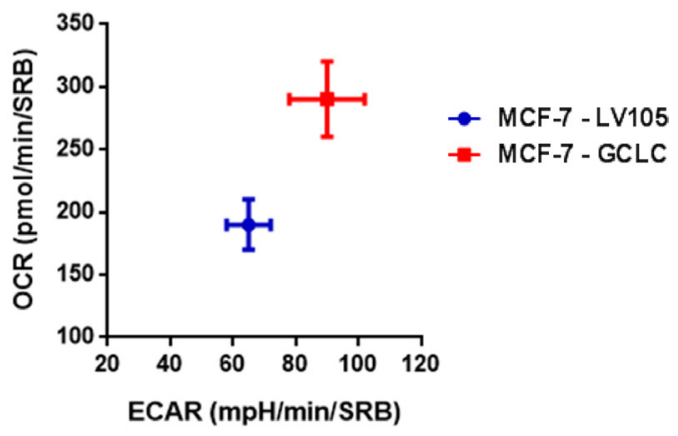

Figure 14: MCF7 cells harboring GCLC are more energetically active, and show a minor increase in their glycolytic rate. The Seahorse XF96 analyzer was employed to determine the status of extracellular acidification rate (ECAR) in MCF-7-LV105 (empty vector) and MCF-7-GCLC cells after 48 hours. A bar graph of 3 independent experiments is shown. B. The plot of OCR versus ECAR shows that MCF-7-TAMR cells shift from moderate quiescent state to a high energetic state. ${ }^{*} p<0.05$.

A

ATP

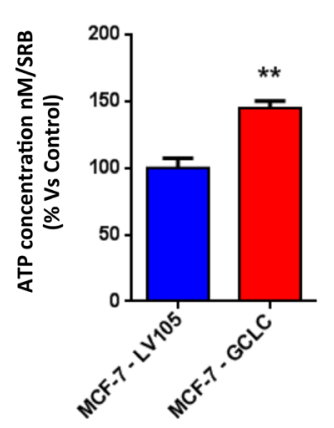

B

GSH/GSSH

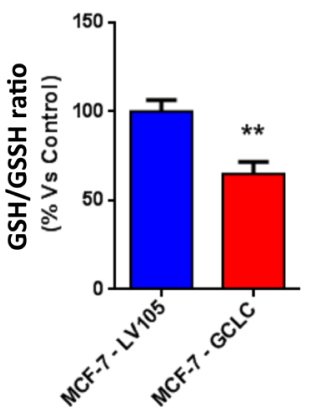

Figure 15: MCF7 cells harboring GCLC are characterized by increased steady-state levels of ATP and decreased levels of reduced glutathione. A. ATP levels evaluated with the Celltiter-Glo ${ }^{\mathrm{TM}}$ luminescent assay kit, after 24 hours of incubation at $37^{\circ} \mathrm{C}$. B. The reduced/oxidized glutathione ratio was evaluated with the GSH/GSSG-Glo ${ }^{\mathrm{TM}}$ Assay kit, after 24 hours of incubation at $37^{\circ} \mathrm{C}$. Both assays were normalized by protein content (SRB) and cell number. $* * p<0.005$. 
similar regulation of numerous cellular pathways (Figure 17B). Importantly, the Heat-Map shows how the different cell lines behave almost in the same way, emphasizing their similarities also in the regulation of numerous cancerrelated signaling pathways. Thus, our proteomics analysis clearly detects alterations in mitochondrial function, the Nrf-2-mediated oxidative stress response and HypoxiaInducible factor (HIF) signaling, all mediated by NQO1 and GCLC as compared with MCF-7-TAMR cells (Figure 18).

\section{Bioinformatic validation of the clinical relevance of NQO1 and GCLC in human breast cancer patients, in various ER(+) epithelial sub-types}

To assess the possible clinical relevance of NQO1 and GCLC, we determined if their mRNA levels showed any prognostic value in human breast cancer patient cohorts, with long-term follow-up (nearly 20 years). The prognostic value of NQO2 was also evaluated in parallel, for comparison purposes. We restricted our analysis to $\mathrm{ER}(+)$ patients that received endocrine therapy (mostly tamoxifen), but not any form of chemotherapy.

These results are summarized in Table 4 , and in Supplementary Tables S1 and S2. Corresponding KaplanMeier (K-M) analysis curves are included in Figure 19, and as Supplementary Figures 4 and 5 (panels A-D).

Note that high mRNA expression levels of all three distinct NQO1 probes showed an association with reduced relapse-free survival (RFS, i.e., higher tumor recurrence). More specifically, NQO1 had prognostic value in Luminal A patients with lymph node metastasis $(\mathrm{LN}(+))$, as well in Luminal B patients, and in the total $\mathrm{ER}(+)$ patient population. GCLC only showed prognostic value in

\section{Mitotracker Deep Red and Orange in GCLC and Control cells in complete media $\mathbf{7 2 h}$}

A

Mitotracker Deep Red

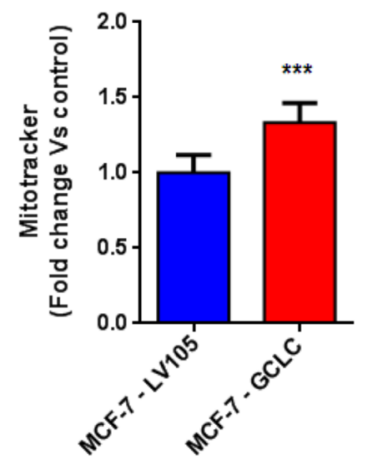

B

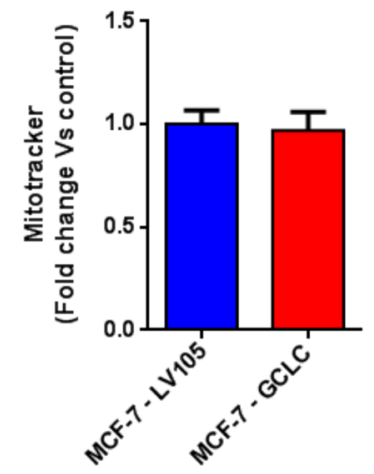

Mitotracker Deep Red and Orange in GCLC Vs Control cells in 4-OHT media 72h

C

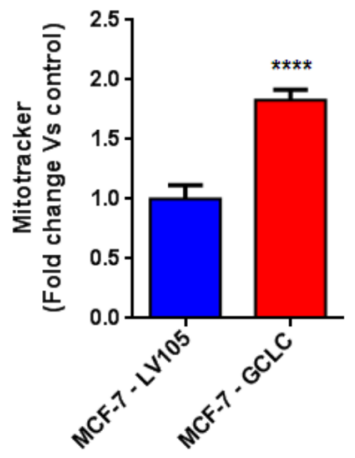

D Mitotracker Orange

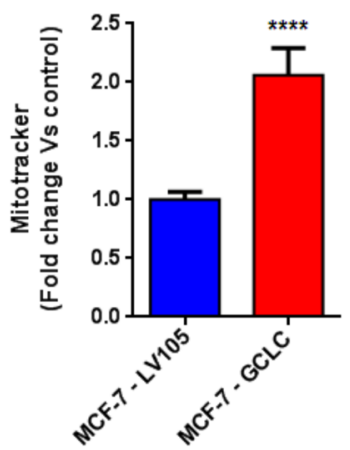

Figure 16: Mitochondrial biogenesis and membrane potential are increased in MCF7 cells harboring GCLC, in the presence of tamoxifen. FACS analysis was carried out on MCF-7-LV105 (empty vector) and MCF-7-GCLC after 72 hours. A.-B. In MCF-7-GCLC, the mitochondrial mass was increased (MitoTracker Deep-Red), but no significant change in mitochondrial membrane potential (MitoTracker Orange) was observed after 72 hours of incubation, in growth media 4-OHT free. C.-D. Both the mitochondrial mass (MitoTracker Deep-Red) and mitochondrial membrane potential (MitoTracker Orange) were highly increased after 48 hours of incubation, in growth media containing 4-OHT. *** $p<0.0001 ; * * * * 0.00001$. 
A

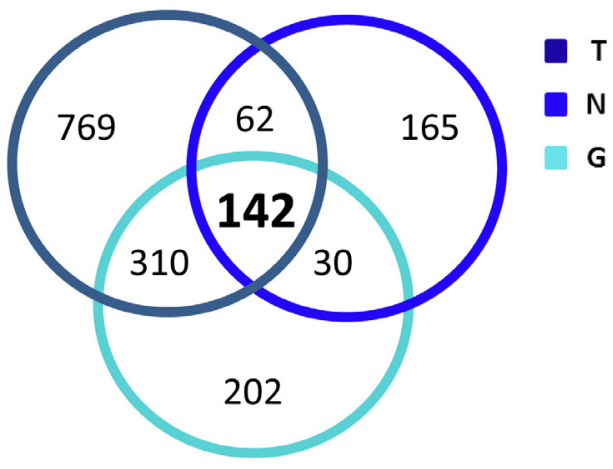

B Canonical Pathways

\begin{tabular}{l} 
Activation z-score \\
\hline \multicolumn{1}{c}{ RhoA Signaling } \\
Signaling by Rho Family GTPases \\
Rac Signaling \\
Leukocyte Extravasation Signaling \\
Integrin Signaling \\
Ephrin B Signaling \\
mTOR Signaling \\
Paxillin Signaling \\
Death Receptor Signaling \\
RhoGDI Signaling \\
ILK Signaling \\
Actin Cytoskeleton Signaling \\
NRF2-mediated Oxidative Stress Response \\
ERK/MAPK Signaling \\
PI3K/AKT Signaling \\
VEGF Signaling \\
Regulation of Actin-based Motility by Rho \\
Agrin Interactions at Neuromuscular Junction \\
Remodeling of Epithelial Adherens Junctions \\
IGF-1 Signaling \\
Aldosterone Signaling in Epithelial Cells \\
CDK5 Signaling \\
PAK Signaling \\
Calcium Signaling \\
AMPK Signaling \\
\hline
\end{tabular}

Figure 17: Overview of proteins and pathways identified as differentially regulated in the lysates of TAMR, NQO1 and GCLC cells relative to control MCF7 cells, by Ingenuity Pathway Analysis. A. Venn diagram. Overlap of differentially regulated proteins identified in MCF-7-TAMR, MCF-7-NQO1 and MCF-7-GCLC, compared with their proper controls. Of all the proteins identified by quantitative proteomics, 142 were proteins that the expression of which was found to be altered in both treatments, compared to control. B. Canonical pathways identified or predicted as altered in MCF-7-TAMR, MCF-7-NQO1 and MCF-7-GCLC, relative to control. A positive $\mathrm{z}$ score is indicated in orange and points towards an activation of the pathway, and a negative $\mathrm{z}$ score, in blue, indicates an inhibition of the pathway. ( $\mathrm{T}=$ MCF-7-TAMR vs. MCF-7-control; N = MCF-7-NQO1 vs. MCF-7-LV105; G = MCF-7-GCLC vs. MCF7-LV105).

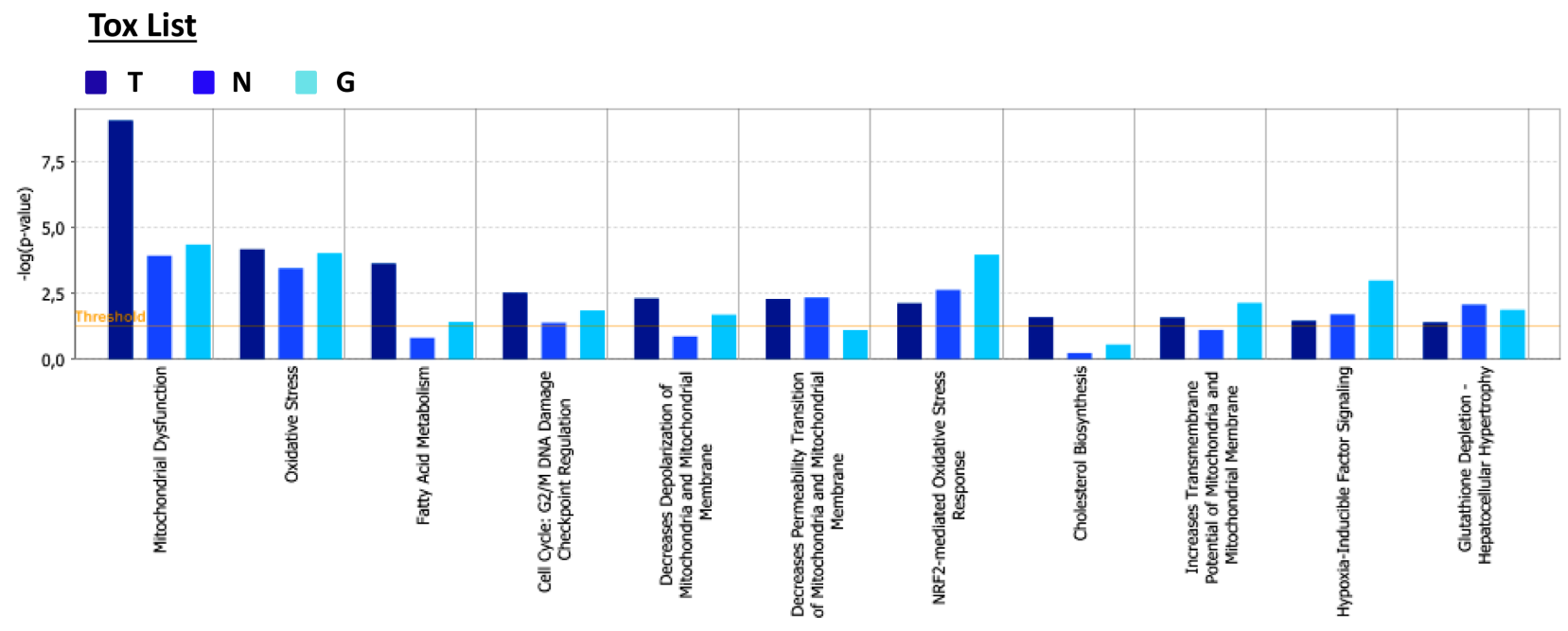

Figure 18: Tox list of pathways identified as differentially regulated in the lysates of TAMR, NQO1 and GCLC cells relative to control MCF7 cells, by Ingenuity Pathway Analysis. Toxicity effects of differentially expressed proteins in MCF-7TAMR, MCF-7-NQO1 and MCF-7-GCLC compared to control cells. Ingenuity Pathway Analysis showed toxicity functions significantly enriched by the proteins differentially expressed in the comparison analysis $(p<0.05)$. In the Bar chart, the $\mathrm{p}$ value for each pathway is indicated by the bar and is expressed as -1 times the log of the $\mathrm{p}$ value. (T = MCF-7-TAMR Vs MCF-7-control; N = MCF-7-NQO1 VS MCF-7-LV105 (empty vector); G = MCF-7-GCLC Vs MCF-7-LV105 (empty vector)). 
Table 4: Prognostic value of NQO1, NQO2 and GCLC in a high-risk subgroup of ER(+) patients: Luminal A/LN(+)

$\begin{array}{llll}\text { Symbol } & \text { Probe Number } & \text { Hazard Ratio (HR) } & \text { P-Value (LogRank) } \\ & & & \\ \text { NQO1-1 } & \text { 201467_s_at } & 2.30 & 0.0026 \\ \text { NQO1-2 } & \text { 201468_s_at } & 2.59 & 0.0012 \\ \text { NQO1-3 } & \text { 210519_s_at } & 2.15 & 0.0065 \\ \text { NQO2 } & \text { 203814_s_at } & 1.52 & 0.14 \\ \text { GCLC } & \text { 202923_s_at } & 1.49 & 0.17\end{array}$

Recurrence-free survival (RFS) in a high-risk population of breast cancer patients: $\mathrm{ER}(+) /$ Luminal A/LN(+)/EndocrineTx (mostly Tamoxifen)/N=152 patients.

A

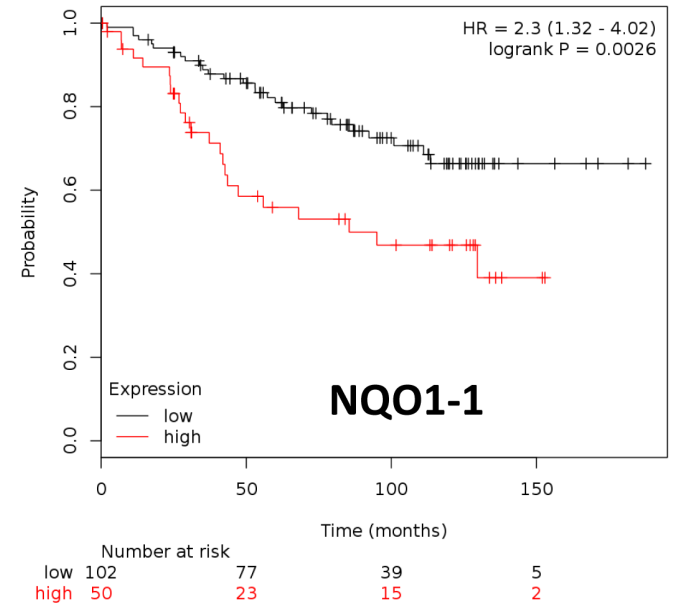

C

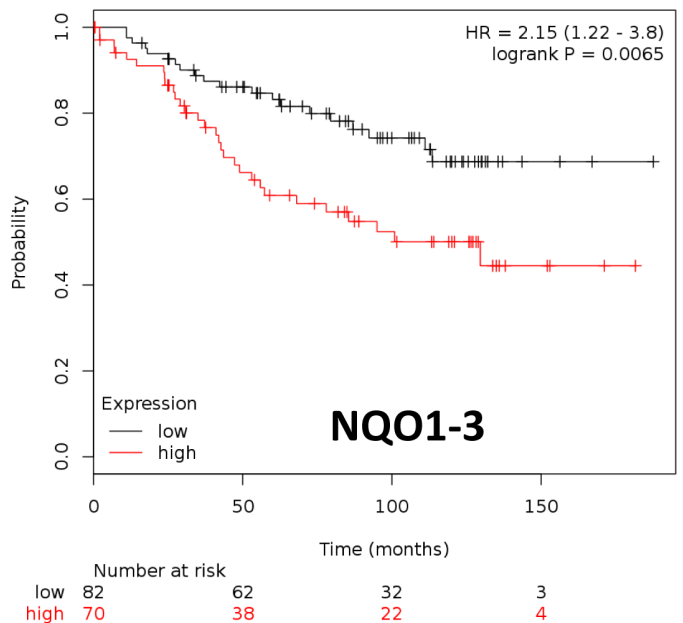

B

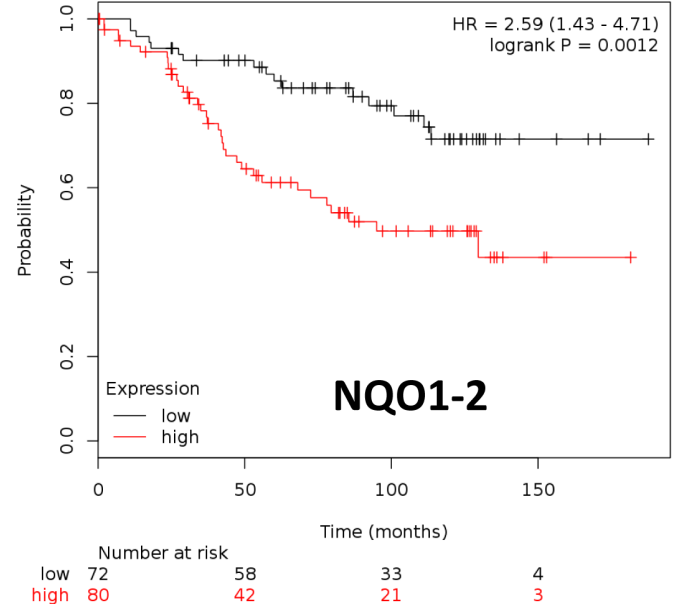

D

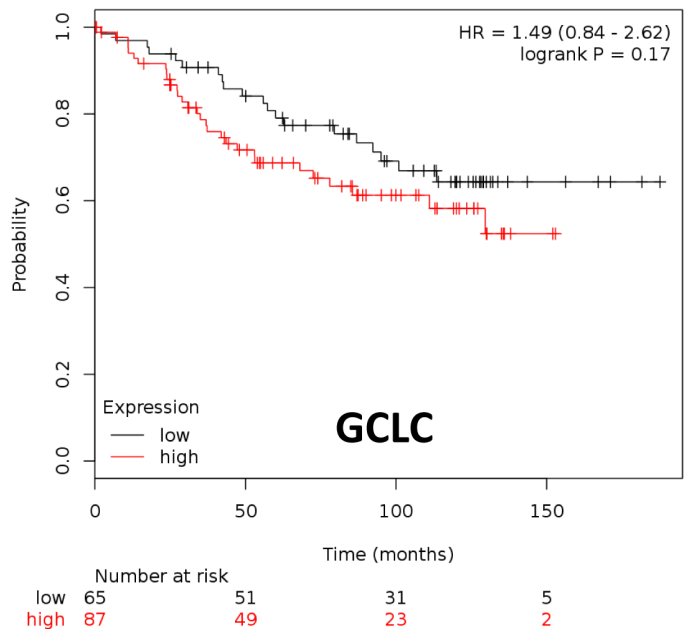

$N=152$

Figure 19: Kaplan-Meier (K-M) analysis of the prognostic value of NQO1 and GCLC in ER(+) breast cancer patients receiving endocrine therapy: Luminal A subgroup. Results of recurrence-free survival analysis (RFS) are shown, over a $>15$-year period of follow-up, for NQO1 (3 independent probes; A.-C., and GCLC D., for ER $(+)$ breast cancer patients $(\mathrm{N}=152)$ with the luminal A sub-type. These patients were lymph-node-positive $(\mathrm{LN}(+))$ and received endocrine therapy (mostly tamoxifen), but not chemotherapy. Note that higher levels of NQO1 mRNA species A.-C. are significantly associated with tumor recurrence. See the Methods section for how the analysis was carried out. Similar results were obtained in other ER $(+)$ sub-types of breast cancer, and are included as Supplementary Figures 4 and 5. 
the Luminal B sub-population of $\mathrm{ER}(+)$ breast cancer patients. In contrast, NQO2 mRNA levels did not show any prognostic value in any of the $\mathrm{ER}(+)$ patient groups examined (Table 4; Supplementary Tables S1 and S2).

Finally, since high levels of NQO1 mRNA were associated with disease progression in patients that received endocrine therapy, this is indicative of a clinical association with endocrine therapy-resistance. Thus, elevated levels of NQO1 expression could be used to identify high-risk ER(+) breast cancer patients, that might benefit from treatment with novel NQO1 inhibitors.

\section{DISCUSSION}

In this report, we set out to identify new metabolic drivers of tamoxifen-resistance, by combining proteomics analysis with metabolic phenotyping [22, 23]. Use of this proteomics-metabolomics approach allowed us to identify enhanced mitochondrial metabolism as a key characteristic of TAMR cells, that was characterized by i) increased oxygen consumption, ii) increased ATP production, and iii) augmented mitochondrial biogenesis, as well as increased oxidative stress, as revealed by iv) decreased levels of reduced glutathione levels (Figure 20). Thus, we focused the interpretation of our proteomics results on key metabolic enzymes that were dramatically increased in tamoxifen-resistant cells, such as NQO1 and GCLC. To determine if the over-expression of NQO1

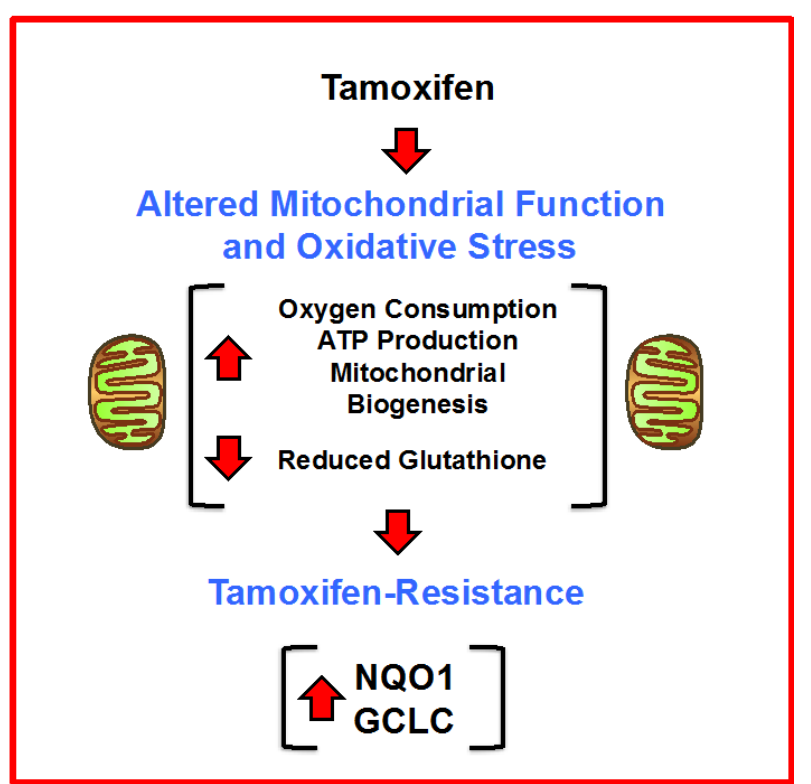

Figure 20: Enhanced mitochondrial metabolism: A new mechanism for driving tamoxifen-resistance. A schematic diagram is shown high-lighting our observations that increased mitochondrial oxygen-consumption and ATPproduction, as well as increased mitochondrial biogenesis and oxidative stress, all contribute to the metabolic phenotype of tamoxifen-resistant breast cancer cells. In this process, the overexpression of NQO1 and GCLC appear to be key drivers of this phenotype. and/or GCLC could autonomously confer the tamoxifenresistance phenotype, we transduced MCF-7 cells with the cDNAs encoding these proteins, using a lenti-viral vector approach. Remarkably, NQO1 and GCLC lenti-viral transduction was indeed sufficient to induce tamoxifenresistance, as well as the metabolically-enhanced mitochondrial phenotype.

Thus, increased mitochondrial function may help drive tamoxifen-resistance in human breast cancer cells (Figure 20). This key finding has important mechanistic implications for understanding the molecular basis of tamoxifen-resistance. Furthermore, the over-expression of NQO1 mRNA species predicted tumor recurrence in highrisk $\mathrm{ER}(+)$ that were $\mathrm{LN}(+)$ and had received endocrinetherapy (mostly tamoxifen), but not chemotherapy. As such, our findings may have translational and clinical relevance for over-coming the resistance to endocrine therapy in $\mathrm{ER}(+)$ breast cancer patients [24].

It remains unknown exactly how NQO1 and GCLC drive an enhanced mitochondrial metabolic phenotype. The NQO1 protein product is an enzyme known as $\mathrm{NAD}(\mathrm{P}) \mathrm{H}$ dehydrogenase [quinone] 1 . It is the main nonmitochondrial enzyme responsible for the reduction of CoQ10 in cells. Thus, NQO1 may "boost" mitochondrial metabolism by providing increased reduced CoQ10 species to facilitate oxidative mitochondrial metabolism $[25,26]$.

The GCLC protein product is an enzyme known as glutamate-cysteine ligase catalytic subunit. Glutamatecysteine ligase is also known as gamma-glutamylcysteine synthetase, and is the first and rate-limiting step for glutathione synthesis. While it remains unknown how GCLC regulates mitochondrial function, its deletion in mouse liver leads to striking decreases in mitochondrial function, mitochondrial injury, loss of cellular ATP, and a marked increase in lipid peroxidation, driving steatosis and liver failure. Interestingly, treatment of these mice with oral N-acetyl-cysteine (NAC) restores mitochondrial function and prevents mortality caused by the loss of hepatocyte GSH synthesis $[27,28]$.

NQO1 and GCLC are normally up-regulated as part of the Nrf2-anti-oxidant response, which occurs during oxidative stress $[12,24]$. Thus, the observed up-regulation of NQO1 and GCLC in TAMR cells may directly reflect that tamoxifen treatment can induce oxidative stress. How does tamoxifen induce oxidative stress? Several different mechanisms have been proposed to explain the manner in which tamoxifen induces oxidative stress in cells, including the targeting of NOS, SOD2 and ER-beta in mitochondria.

Tamoxifen also has direct effects on mitochondrial function. Tamoxifen acts both as an uncoupling agent and as a potent inhibitor of electron transport, ultimately leading to a collapse of the mitochondrial membrane potential and cellular apoptosis [29-31]. More specifically, tamoxifen inhibits electron transfer at the levels of both 
complex III (ubiquinol-cytochrome-c reductase) and complex IV (cytochrome-c oxidase). Similarly, it has also been reported that tamoxifen elevates mitochondrial ROS, mitochondrial lipid peroxidation and cytochrome c release, while driving the tyrosine-nitration of key mitochondrial proteins.

Therefore, our current findings could mechanistically explain why "boosting" mitochondrial metabolism may lead to tamoxifen-resistance in human breast cancer cells. If tamoxifen indeed behaves as a mitochondrial "poison", then increased mitochondrial "power" could help to buffer against the mitochondrial toxicity effects of tamoxifen, thereby conferring tamoxifen-resistance.

\section{MATERIALS AND METHODS}

\section{Materials}

MCF7 cells (catalogue \# HTB-22), a human breast cancer cell line, were originally obtained from the ATCC cell repository. Tamoxifen and dicoumarol were obtained commercially from Sigma-Aldrich, Inc.

\section{Label-free semi-quantitative proteomics analysis}

Cell lysates were prepared for trypsin digestion by sequential reduction of disulphide bonds with TCEP and alkylation with MMTS. Then, the peptides were extracted and prepared for LC-MS/MS. All LC-MS/MS analyses were performed on an LTQ Orbitrap XL mass spectrometer (Thermo Scientific, San Jose, CA) coupled to an Ultimate 3000 RSLCnano system (Thermo Scientific, formerly Dionex, The Netherlands). Xcalibur raw data files acquired on the LTQ-Orbitrap XL were directly imported into Progenesis LCMS software (Waters Corp) for peak detection and alignment. Data were analyzed using the Mascot search engine. Five technical replicates were analyzed for each sample type [32].

\section{Ingenuity pathway analyses}

Pathway and function analyses were generated using Ingenuity Pathway Analysis (IPA) (Ingenuity systems, http://www.ingenuity.com), which assists with proteomics data interpretation via grouping differentially expressed genes or proteins into known functions and pathways. Pathways with a $\mathrm{z}$ score $>1.5$ were considered as significantly activated, and pathways with a $\mathrm{z}$ score $<$ -1.5 were considered as significantly inhibited.

\section{Lentiviral gene transduction}

Lentiviral plasmids, packaging cells and reagents were purchased from Genecopoeia. 48 hours after seeding, 293Ta packaging cells were transfected with lentiviral vectors encoding NQO1 or GCLC or empty vector (EX-NEG-Lv105), using Lenti-PacTM HIV Expression Packaging Kit according to the manufacturer's instructions. Two days post-transfection, lentiviruscontaining culture medium was passed through a $0.45 \mu \mathrm{m}$ filter and added to the target cells (MCF-7 cells) in the presence of $5 \mu \mathrm{g} / \mathrm{ml}$ Polybrene. Infected cells were selected with a concentration of $1.5 \mu \mathrm{g} / \mathrm{ml}$ of puromycin.

\section{Cellular growth using the sulforhodamine B (SRB) assay}

SRB (S9012, Sigma) measures total biomass by staining cellular proteins. After 2, 5 and 8 days, cells were fixed in $10 \%$ trichloroacetic acid (T9159, Sigma) for $1 \mathrm{~h}$ at $4^{\circ} \mathrm{C}$, stained with SRB (S9012, Sigma) for 15 minutes, and washed 3 times with $1 \%$ acetic acid (27225, Sigma). The incorporated die was solubilized with 10 mM Tris Base, $\mathrm{pH} 8.8$ (T1503, Sigma). Absorbance was spectrophotometrically measured at $540 \mathrm{~nm}$ in a FluoStar Omega plate reader (BMG Labtech). Background measurements were subtracted from all values. Dicoumarol was obtained from Sigma-Aldrich, Inc.

\section{Seahorse XF96 metabolic flux analysis (OCR and ECAR)}

Real-time oxygen consumption rates (OCR) and extracellular acidification rates (ECAR) measurements were determined using the Seahorse Extracellular Flux (XF96) analyzer (Seahorse Bioscience, MA, USA). $1 \times 10^{4}$ cells per well were seeded into XFe-96 well cell culture plates, and incubated overnight to allow attachment. Control cells were processed in parallel. After 48 hours of incubation, cells were washed in pre-warmed $\mathrm{XF}$ assay media (or for OCR measurement, XF assay media supplemented with $10 \mathrm{mM}$ glucose, $1 \mathrm{mM}$ Pyruvate, $2 \mathrm{mM}$ L-glutamine and adjusted at $7.4 \mathrm{pH}$ ). Cells were then maintained in $175 \mu \mathrm{L} /$ well of XF assay media at $37^{\circ} \mathrm{C}$, in a non- $\mathrm{CO}_{2}$ incubator for 1 hour. During the incubation time, we loaded $25 \mu \mathrm{L}$ of $80 \mathrm{mM}$ glucose, $9 \mu \mathrm{M}$ oligomycin, and $1 \mathrm{M}$ 2-deoxyglucose (for ECAR measurement) or $10 \mu \mathrm{M}$ oligomycin, $9 \mu \mathrm{M}$ FCCP, $10 \mu \mathrm{M}$ rotenone, $10 \mu \mathrm{M}$ antimycin A (for OCR measurement), in XF assay media into the injection ports in the XFe-96 sensor cartridge. Data sets were analyzed by XF96 software and GraphPad Prism software, using one-way ANOVA and Student's $t$-test calculations. All experiments were performed in quintuplicate, three times independently. 


\section{ATP and glutathione levels}

Cell activity was measured using a CellTiter-Glo ${ }^{\mathrm{TM}}$ assay kit (Promega, Madison, WI \#G7571) and GSH/ GSSG-Glo ${ }^{\mathrm{TM}}$ Assay kit (Promega, Madison, WI \#V6612) following the manufacturer's protocol. For ATP detection, $2 \times 10^{4}$ cells were seeded in a 96-well plate and incubated for $24 \mathrm{~h}$. After 24 hours, Celltiter-Glo reagent was added to each well, and the plate was incubated at room temperature for 10 minutes with constant shaking before detected the luminescence intensity. For GSH/GSSH ratio content, 1 x $10^{4}$ cells were seeded in a 96-well plate and incubated for $24 \mathrm{~h}$. After 24 hours, Total and Oxidized Glutathione reagents were added to each well and incubated for 30 minutes. Luciferin was added to each well and the plate was read after 15 minutes. Luminescence intensity was read using a FluoStar Omega plate reader (BMG Labtech) taking the luminescence reading of control as the $100 \%$ value. Background measurements were subtracted from all values. The experiments were performed using $4 \times 10^{4}$ cells also and 48 hours of incubation. The results followed the same trend (data not shown). All experiments were performed in sixtuplicate, four times independently.

\section{Mitochondrial staining}

Mitochondrial activity was assessed with MitoTracker Orange (\#M7510, Invitrogen), whose accumulation in mitochondria isdependent upon membrane potential. Mitochondrial mass was determined using MitoTracker Deep-Red (\#M22426, Invitrogen), localizing to mitochondria regardless of mitochondrial membrane potential. $2 \times 10^{5}$ cells were seeded in a 6 well plate and leave at $37^{\circ} \mathrm{C}$ for 72 hours. Control cells were processed in parallel. After 72 hours, cells were incubated with prewarmed MitoTracker staining solution (diluted in PBS/CM to a final concentration of $10 \mathrm{nM}$ ) for $30-60$ minutes at $37^{\circ} \mathrm{C}$. All subsequent steps were performed in the dark. Cells were washed in PBS, harvested, and re-suspended in $300 \mu \mathrm{L}$ of $\mathrm{PBS} / \mathrm{CM}$. Cells were then analyzed by flow cytometry. Data analysis was performed using FlowJo software.

\section{Statistical analysis}

Data is represented as the mean \pm standard deviation (SD), taken over $\geq 3$ independent experiments, with $\geq 3$ technical replicates per experiment, unless otherwise stated. Statistical significance was measured using the analysis of variance (ANOVA) test or student $t$-test. $P \leq$ 0.05 was considered significant and all statistical tests were two sided. Mitochondrial staining dats are represented as the mean \pm standard deviation (SEM), taken over $\geq 3$ independent experiments, with $\geq 3$ technical replicates per experiment, unless otherwise stated. Statistical significance was measured using the analysis of variance (ANOVA) test. $P \leq 0.05$ was considered significant and all statistical tests were two sided.

\section{Kaplan-Meier (K-M) analysis}

To perform K-M analysis on NQO1, NQO2 and GCLC gene transcripts, we used an open-access online survival analysis tool to interrogate publically available microarray data from $>5,000$ breast cancer patients. This allowed us to determine their prognostic value. For this purpose, we primarily analyzed data from $\operatorname{ER}(+)$ patients that were $\mathrm{LN}(+)$ at diagnosis and were of the luminal A sub-type, that were primarily treated with tamoxifen and not other chemotherapy ( $N=152$ patients). Biased and outlier array data were excluded from the analysis. Hazard-ratios were calculated, at the best auto-selected cut-off, and p-values were calculated using the logrank test and plotted in R. K-M curves were also generated online using the K-M-plotter (as high-resolution TIFF files), using univariate analysis: http://kmplot.com/analysis/ index.php? $p=$ service\&cancer $=$ breast. This allowed us to directly perform in silico validation of these biomarker candidates. The most updated version of the database was utilized for all these analyses.

\section{Author contributions}

Professor Lisanti conceived and initiated this collaborative project. All the experiments in this paper were designed and performed by Marco Fiorillo, with minor technical assistance from other lab members. Marco Fiorillo analyzed all the data and generated the final figures and tables, and he wrote significant portions of the manuscript. Professors Lisanti, Sotgia, Sisci and Cappello all contributed to the writing and the editing of the manuscript. Professor Lisanti performed the K-M analysis online, using a publically available database, and generated the schematic summary diagram. Authorship decisions were reached as a general consensus between all the co-authors.

\section{ACKNOWLEDGMENTS}

The authors would like to thank Hannah Harrison for her technical help with cell propagation and for the submission of samples to the proteomics facility. Similarly, we would also like to thank Amy Chadwick for her technical assistance with the following laboratory procedures: lentiviral transduction, training on the Seahorse, advice on graphical representation (especially with GraphPad Software) and for the submission of samples to the proteomics facility. We are grateful to Duncan Smith, who performed the proteomics analysis 
on whole cell lysates, within the CRUK Core Facility. We also thank Robert Clarke for generously donating the TAMR cells. TAMR cells were used initially for our early studies, but further validation was also carried out with three other tamoxifen-resistant cell lines (MCF-7NQO1 and MCF-7-GCLC cells, as well as TAMR2 cells). TAMR2 cells were provided by Professor Diego Sisci. The Sotgia and Lisanti Laboratories are currently supported by private donations, the Healthy Life Foundation and by funds from the University of Salford. This study was also supported by a Grant IG 15738/2014 from the Associazione Italiana Ricerca sul Cancro (AIRC).

\section{CONFLICTS OF INTEREST}

There is no conflict of interest.

\section{REFERENCES}

1. Ali S, Coombes RC. Endocrine-responsive breast cancer and strategies for combating resistance. Nature Reviews Cancer. 2002; 2: 101-112.

2. Osborneand CK, Schiff R. Mechanisms of endocrine resistance in breast cancer. Annual Review of Medicine. 2011; 62: 233-247.

3. Li X, Lewis MT, Huang J, Gutierrez C, Osborne CK, Wu MF, Hilsenbeck SG, Pavlick A, Zhang X, Chamness GC, Wong H, Rosen J, Chang JC. Intrinsic resistance of tumorigenic breast cancer cells to chemotherapy. Journal of the National Cancer Institute. 2008; 100: 672-679.

4. Clarke R, Tyson JJ, Dixon JM. Endocrine resistance in breast cancer--An overview and update. Molecular and cellular Endocrinology. 2015; 418 :220-234.

5. Giuliano M, Schifp R, Osborne CK, Trivedi MV. Biological mechanisms and clinical implications of endocrine resistance in breast cancer. Breast. 2011; 20: S4249.

6. Dittmer J, Leyh B. The impact of tumor stroma on drug response in breast cancer. Seminars in Cancer Biology. 2015; 31:3-15.

7. Jordan VC. Selective estrogen receptor modulation: concept and consequences in cancer. Cancer Cell. 2004; 5: 207-213.

8. Martinez-Outschoorn UE, Goldberg A, Lin Z, Ko YH, Flomenberg N, Wang C, Pavlides S, Pestell RG, Howell A, Sotgia F, Lisanti MP. Anti-estrogen resistance in breast cancer is induced by the tumor microenvironment and can be overcome by inhibiting mitochondrial function in epithelial cancer cells. Cancer Biology \& Therapy. 2011; 12:924-938.

9. Onodera Y, Motohashi H, Takagi K, Miki Y, Shibahara Y, Watanabe M, Ishida T, Hirakawa H, Sasano H, Yamamoto M, Suzuki T. NRF2 immunolocalization in human breast cancer patients as a prognostic factor. Endocrine-related Cancer. 2014; 21: 241-252.
10. Li J, Shin S, Sun Y, Yoon SO, Li C, Zhang E, Yu J, Zhang J, Blenis J. mTORC1-Driven Tumor Cells Are Highly Sensitive to Therapeutic Targeting by Antagonists of Oxidative Stress. Cancer Research. 2016; 76: 4816-4827.

11. Syu JP, Chi JT, Kung HN. Nrf2 is the key to chemotherapy resistance in MCF7 breast cancer cells under hypoxia. Oncotarget. 2016; 7:14659-14672. doi: 10.18632/ oncotarget.7406.

12. DeNicola GM, Karreth FA, Humpton TJ, Gopinathan A, Wei C, Frese K, Mangal D, Yu KH, Yeo CJ, Calhoun ES, Scrimieri F, Winter JM, Hruban RH, et al. Oncogeneinduced Nrf2 transcription promotes ROS detoxification and tumorigenesis. Nature. 2011; 475:106-109.

13. Glorieux C, Sandoval JM, Dejeans N, Ameye G, Poirel HA, Verrax J, Calderon PB. Overexpression of NAD(P) $\mathrm{H}$ :quinone oxidoreductase 1 (NQO1) and genomic gain of the NQO1 locus modulates breast cancer cell sensitivity to quinones. Life sciences. 2016; 145: 57-65.

14. Yang Y, Zhang Y, Wu Q, Cui X, Lin Z, Liu S, Chen L. Clinical implications of high NQO1 expression in breast cancers. J Exp Clin Cancer Res. 2014; 33: 14.

15. Zanotto-Filho A, Masamsetti VP, Loranc E, Tonapi SS, Gorthi A, Bernard X, Gonçalves RM, Moreira JC, Chen Y, Bishop AJ. Alkylating agent induced NRF2 blocks endoplasmic reticulum stress-mediated apoptosis via control of glutathione pools and protein thiol homeostasis. Mol Cancer Ther. 2016; 15: 3000-3014.

16. Knowlden JM, Hutcheson IR, Jones HE, Madden T, Gee JM, Harper ME, Barrow D, Wakeling AE, Nicholson RI. Elevated levels of epidermal growth factor receptor/ c-erbB2 heterodimers mediate an autocrine growth regulatory pathway in tamoxifen-resistant MCF-7 cells. Endocrinology. 2003; 144: 1032-1044.

17. Fiorillo M, Lamb R, Tanowitz HB, Mutti L, KrsticDemonacos M, Cappello AR, Martinez-Outschoorn UE, Sotgia F, Lisanti MP. Repurposing atovaquone: targeting mitochondrial complex III and OXPHOS to eradicate cancer stem cells. Oncotarget. 2016; 7: 34084-34099. doi: 10.18632/oncotarget.9122.

18. De Luca A, Fiorillo M, Peiris-Pagès M, Ozsvari B, Smith DL, Sanchez-Alvarez R, Martinez-Outschoorn UE, Cappello AR, Pezzi V, Lisanti MP, Sotgia F. Mitochondrial biogenesis is required for the anchorageindependent survival and propagation of stem-like cancer cells. Oncotarget. 2015; 6: 14777-14795. doi: 10.18632/ oncotarget.4401.

19. Fiorillo M, Lamb R, Tanowitz HB, Cappello AR, MartinezOutschoorn UE, Sotgia F, Lisanti MP. Bedaquiline, an FDA-approved antibiotic, inhibits mitochondrial function and potently blocks the proliferative expansion of stem-like cancer cells (CSCs). Aging (Albany NY). 2016; 8: 1593 1607. doi: 10.18632/aging.100983.

20. Casaburi I, Avena P, De Luca A, Chimento A, Sirianni R, Malivindi R, Rago V, Fiorillo M, Domanico F, Campana C, Cappello AR, Sotgia F, Lisanti MP, et al. Estrogen 
related receptor alpha (ERRalpha) a promising target for the therapy of adrenocortical carcinoma (ACC). Oncotarget. 2015; 6: 25135-25148. doi: 10.18632/oncotarget.4722.

21. Peiris-Pagès M, Smith DL, Győrffy B, Sotgia F, Lisanti MP. Proteomic identification of prognostic tumour biomarkers, using chemotherapy-induced cancer-associated fibroblasts. Aging (Albany NY). 2015; 7: 816-838.doi: 10.18632/ aging. 100808 .

22. Shajahan-Haq AN, Cheema MS, Clarke R. Application of metabolomics in drug resistant breast cancer research. Metabolites. 2015; 5: 100-118.

23. Martinez-Outschoorn UE, Peiris-Pagés M, Pestell RG, Sotgia F, Lisanti MP. Cancer metabolism: a therapeutic perspective. Nature Reviews Clinical Oncology. 2017; 14: 11-31.

24. Bekele RT, Venkatraman G, Liu RZ, Tang X, Mi S, Benesch MG, Mackey JR, Godbout R, Curtis JM, McMullen TP, Brindley DN. Oxidative stress contributes to the tamoxifen-induced killing of breast cancer cells: implications for tamoxifen therapy and resistance. Scientific Reports. 2016; 6: 21164.

25. Li JZ, Ke Y, Misra HP, Trush MA, Li YR, Zhu H, Jia $Z$. Mechanistic studies of cancer cell mitochondria- and NQO1-mediated redox activation of beta-lapachone, a potentially novel anticancer agent. Toxicol Appl Pharmacol. 2014; 281: 285-293.

26. Gueven N, Woolley K, Smith J. Border between natural product and drug: Comparison of the related benzoquinones idebenone and coenzyme Q10. Redox Biology. 2015; 4: 289-295.

27. Chen Y, Johansson E, Yang Y, Miller ML, Shen D, Orlicky DJ, Shertzer HG, Vasiliou V, Nebert DW, Dalton TP. Oral N-acetylcysteine rescues lethality of hepatocytespecific Gclc-knockout mice, providing a model for hepatic cirrhosis. Journal of Hepatology. 2010; 53: 1085-1094.

28. Chen Y, Yang Y, Miller ML, Shen D, Shertzer HG, Stringer KF, Wang B, Schneider SN, Nebert DW, Dalton TP. Hepatocyte-specific Gclc deletion leads to rapid onset of steatosis with mitochondrial injury and liver failure. Hepatology. 2007; 45: 1118-1128.

29. Nazarewicz RR, Zenebe WJ, Parihar A, Larson SK, Alidema E, Choi J, Ghafourifar P. Tamoxifen induces oxidative stress and mitochondrial apoptosis via stimulating mitochondrial nitric oxide synthase. Cancer Research. 2007; 67:1282-1290.

30. Razandi M, Pedram A, Jordan VC, Fuqua S, Levin ER. Tamoxifen regulates cell fate through mitochondrial estrogen receptor beta in breast cancer. Oncogene. 2013; 32: 3274-3285.

31. Tuquet C, Dupont J, Mesneau A, Roussaux J. Effects of tamoxifen on the electron transport chain of isolated rat liver mitochondria. Cell Biology and Toxicology. 2000; 16: 207-219.

32. Lamb R, Fiorillo M, Chadwick A, Ozsvari B, Reeves KJ,
Smith DL, Clarke RB, Howell SJ, Cappello AR, MartinezOutschoorn UE, Peiris-Pagès M, Sotgia F, Lisanti MP. Doxycycline down-regulates DNA-PK and radiosensitizes tumor initiating cells: Implications for more effective radiation therapy. Oncotarget. 2015; 6: 14005-14025. doi: 10.18632/oncotarget.4159. 